\title{
Antiepileptic drug treatment of generalised tonic-clonic seizures: an evaluation of regulatory data and five criteria for drug selection
}

Authors: Professor Simon D Shorvon, ${ }^{1}$ Professor Pedro E Bermejo, ${ }^{2}$ Dr Ayana A Gibbs, ${ }^{3}$ Dr Gilles Huberfeld, ${ }^{4}$ Professor Reetta Kälviäinen ${ }^{5}$

${ }^{1}$ UCL Institute of Neurology, National Hospital for Neurology and Neurosurgery.

London WC1N 3BG, UK; ${ }^{2}$ Hospital Universitario Puerta de Hierro-Majadahonda, Majadahonda, Spain; ${ }^{3}$ Eisai Europe Ltd, Hatfield, UK; ${ }^{4}$ Sorbonne Université, PitiéSalpêtrière Hospital, Neurophysiology Department, Paris, France; and INSERM U1129 "Infantile Epilepsies and Brain Plasticity", Paris Descartes University, PRES Sorbonne Paris Cité, Paris, France; ${ }^{5}$ Epilepsy center/Neurocenter, Kuopio University Hospital and Faculty of Health Sciences, School of Medicine, Institute of Clinical Medicine, University of Eastern Finland, Kuopio, Finland

Corresponding author: Professor Simon Shorvon,

s.shorvon@ucl.ac.uk, Tel.: 0207676 2156, Fax: 02076762155

Word count: $\sim 5,748.6$ Tables. [5 Supplementary tables]

Running title: AEDs in generalised tonic-clonic seizures

Keywords: antiepileptic drugs; generalised seizures; efficacy; tonic-clonic seizures; adverse events 


\section{Supporting Information (online only)}

Additional supporting information can be found in the online version of this article:

Supplementary Table S-1. Rare and idiosyncratic reactions (from the SPC Special Warnings and Precautions section of AEDs approved for use in both PGTCS and SGTCS)

Supplementary Table S-2. Risk, and recommendations for use, in pregnancy (from the SPCs of AEDs approved for use in both PGTCS and SGTCS)

Supplementary Table S-3. Metabolic route, and induction/inhibition of hepatic enzymes (from the SPCs of AEDs approved for use in both PGTCS and SGTCS)

Supplementary Table S-4a. Interactions between AEDs and other drugs - impact of drugs on AED levels (from the SPCs of AEDs approved for both PGTCS and SGTCS)

Supplementary Table S-4b. Interactions between AEDs and other drugs - impact of AEDs on levels of other medicinal products (from the SPCs of AEDs approved for both PGTCS and SGTCS) 


\begin{abstract}
BACKGROUND: A generalised tonic-clonic seizure (GTCS) is the most severe form of common epileptic seizure, and carries the greatest risk of harm. The aim of this review is to provide an evidence-based guide for the selection of antiepileptic drugs (AEDs) for patients with GTCS. Eight AEDs are approved in Europe and the USA for the treatment of both primary GTCS (PGTCS) and secondarily GTCS (SGTCS), and are considered in this paper.
\end{abstract}

METHODS: Each AED is evaluated using five criteria: (1) efficacy, by seizure type (a: PGTCS and b: SGTCS); (2) adverse effects; (3) interactions; (4) adherence and dosing; and (5) mechanism of action. To ensure the inclusions of robust data, only efficacy data accepted by regulatory authorities were considered, and data related to adverse effects, interactions, adherence and mechanism of action were all extracted from UK Summaries of Product Characteristics (SPCs).

RESULTS: (1a) There is Class 1 evidence of the efficacy of only four AEDs in controlling PGTCS (lamotrigine, levetiracetam, perampanel, topiramate). (1b) There is no Class 1 evidence of the efficacy of any AED in SGTCS; although some evidence from pooled/subgroup analyses or meta analyses supports the use of four AEDs (levetiracetam, perampanel, topiramate, and with less robust data for lamotrigine). (2) AEDs are associated with different, but to some extent overlapping, common adverse effect profiles, but have differing idiosyncratic adverse effects. (3) Pharmacokinetic interactions are seen with most, but not all, AEDs, and are most common with carbamazepine and phenytoin. (4) Good adherence is important for seizure control, and is influenced by frequency of dosing, among other factors. (5) Mechanism of 
action is also a consideration in rationalising AED selection when switching or combining AEDs.

CONCLUSION: Ultimately, the choice of AED depends on all these factors, but particularly on efficacy and adverse effects. Different patients will weigh the various factors differently, and the role of the treating physician is to provide accurate information to allow patients to make informed choices.

[Abstract word count: 324 


\section{INTRODUCTION}

It is somewhat paradoxical that although there is universal agreement that generalised tonic-clonic seizures (GTCS) are the most severe form of common epileptic seizure, the treatment of GTCS has been the subject of relatively few robust clinical studies.

A specific focus on GTCS is certainly justified. The detrimental effect of these seizures on many aspects of life for patients with epilepsy has been demonstrated in studies worldwide [1-4]. For example, GTCS carry a much greater risk of accidental death (e.g. from drowning, burns or falls), of SUDEP [5-8] and of accidental injury than focal seizures $[9,10]$. GTCS have been associated with cerebral damage and cognitive decline, including risks to memory [11-13]. If prolonged, they form the most dangerous form of status epilepticus and - because of their dramatic and shocking nature - can cause much psychological distress and have negative consequences for education, work, relationships, social interactions and selfconfidence.

Selection of appropriate AEDs for initial treatment, and for switching and combining therapies if seizures persist, is of crucial importance for patients with GTCS. In this review, we evaluate the best available evidence to aid clinicians in selecting AEDs for the treatment of both primary generalised tonic-clonic seizures (PGTCS) which occur in the context of Idiopathic Generalised Epilepsy, and secondarily generalised tonicclonic seizures (SGTCS) which occur in the context of focal epilepsies.

We evaluated available data using five criteria which are, in our view, the most relevant considerations when making an AED choice: (1) efficacy in treating the specific seizure type; (2) adverse effects - including the common side-effects, the 
uncommon but serious idiosyncratic effects, and the potential for teratogenic effects; (3) drug interactions; (4) adherence, which should be optimised to give any treatment the best chance to work; and (5), mechanism of action, which can help to inform appropriate AED selection when switching or when combining drugs.

To avoid bias and provide the best evidence-based evaluation of robust data, we have considered efficacy data only from the randomised double-blinded studies submitted to the regulatory authorities, and other data only from the drug's official Summary of Product Characteristics (SPC), which are documents with data approved by the regulatory authority. (We have used the UK SPCs approved by the European Medicines Agency.) This is a key feature of this review and we are not aware of other papers which have adopted this methodology.

\section{METHODS}

As this study involved a retrospective review of regulatory documents and clinical trial evidence, institutional review board or ethics committee approval were not required. Definitions of levels of evidence we use are consistent with those from the Centre for Evidence Based Medicine [14].

\section{Antiepileptic drugs}

For this review, we considered evidence related to the eight AEDs that are licensed for the treatment of both PGTCS and SGTCS in Europe and the USA (carbamazepine [15,16], clobazam [17], lamotrigine [18], levetiracetam [19], perampanel [20], phenytoin [21], topiramate [22] and valproate [23] (Table 1), according to European Summary of Product Characteristics (SPCs) and US prescribing information (USPI). 
Lamotrigine, levetiracetam, perampanel and topiramate are licensed on the basis of RCT evidence of efficacy (Level 1 evidence). However, the licenses of carbamazepine, clobazam, phenytoin and valproate are simply based on 'grandfather clauses' (i.e. that the drugs were in use before current licensing regulations were formulated) with no Level 1 clinical trials providing robust evidence of their efficacy. The AED phenobarbital is also licensed on a grandfather clause for epilepsy; however, because it is not widely used in Europe or the USA and does not feature in standard guidelines, we did not include it in this review. It is used widely in countries with limited resources but there is no RCT data to support its use. Please note too that, to be rigorous, we have used the terms PGTCS and SGTCS as these were the terms used in the studies and predate the recent update by the ILAE of terminology (eg SGTCS to focal to bilateral tonic-clonic seizures). 
Table 1. Approved indications of the eight antiepileptic drugs licensed for use in primary and secondarily generalised tonic-clonic seizures

\begin{tabular}{|c|c|c|c|c|}
\hline \multirow{2}{*}{$\begin{array}{l}\text { Antiepileptic } \\
\text { drug }\end{array}$} & \multicolumn{2}{|l|}{ Europe } & \multicolumn{2}{|l|}{ USA } \\
\hline & Seizure types & $\begin{array}{l}\text { Adjunctive/ } \\
\text { monotherapy }\end{array}$ & Seizure types & $\begin{array}{l}\text { Adjunctive/ } \\
\text { monotherapy }\end{array}$ \\
\hline Carbamazepine & Generalised tonic-clonic seizures, partial seizures. & Not specified & $\begin{array}{l}\text { Partial seizures, generalised tonic-clonic seizures } \\
\text { (grand mal), and mixed seizure patterns }\end{array}$ & Not specified \\
\hline Clobazam & Epilepsy & Adj & $\begin{array}{l}\text { Seizures associated with LGS in patients } \geq 2 \text { years } \\
\text { of age. }\end{array}$ & Adj \\
\hline Lamotrigine & $\begin{array}{l}\text { In adults and adolescents aged } \geq 13 \text { years: } \\
\text { - Partial seizures and generalised seizures, }{ }^{\mathrm{a}} \text { including } \\
\text { tonic-clonic seizures and seizures } \\
\text { - Seizures associated with LGS } \\
\text { In children and adolescents aged } 2-12 \text { years: } \\
\text { - Partial seizures, generalised seizures, }{ }^{\mathrm{a}} \text { and seizures } \\
\text { associated with LGS } \\
\text { - Typical absence seizures } \\
\end{array}$ & $\begin{array}{l}\text { Adj and Mono } \\
\text { Adj }^{\mathrm{b}} \\
\text { Adj } \\
\text { Mono }\end{array}$ & $\begin{array}{l}\text { In patients aged } \geq 2 \text { years: } \\
\text { - Partial seizures, primary generalised tonic-- } \\
\text { clonic seizures, generalised seizures of LGS } \\
\text { In adults aged } 16 \text { years and receiving treatment } \\
\text { with carbamazepine, phenytoin, phenobarbital, } \\
\text { primidone, or valproate as the single AED } \\
\text { - Partial seizures }\end{array}$ & $\begin{array}{l}\text { Conversion to } \\
\text { Mono }\end{array}$ \\
\hline Levetiracetam & $\begin{array}{l}\text { In adults and adolescents aged } \geq 16 \text { years with newly } \\
\text { diagnosed epilepsy with partial-onset seizures, }{ }^{\mathrm{c}} \\
\text { In patients aged } \geq 1 \text { month } \\
\text { - Partial-onset seizures, } \\
\text { Adults and adolescents } \geq 12 \text { years: } \\
\text { - Myoclonic seizures in JME } \\
\text { - Primary generalised tonic-clonic seizures in IGE }\end{array}$ & $\begin{array}{l}\text { Mono } \\
\text { Adj } \\
\text { Adj } \\
\text { Adj }\end{array}$ & $\begin{array}{l}\text { In patients aged } \geq 6 \text { years: } \\
\text { - } P G T C S \text { in } I G E \\
\text { In patients aged } \geq 1 \text { month: } \\
\text { - Partial-onset seizures } \\
\text { In patients aged } \geq 12 \text { years: } \\
\text { - Myoclonic seizures in JME }\end{array}$ & $\begin{array}{l}\text { Adj } \\
\text { Adj } \\
\text { Adj }\end{array}$ \\
\hline Perampanel & $\begin{array}{l}\text { In adults and adolescents aged } \geq 12 \text { years: } \\
\text { - Partial-onset seizures }{ }^{\mathrm{c}} \\
\text { - PGTCS in IGE }\end{array}$ & $\begin{array}{l}\text { Adj } \\
\text { Adj }\end{array}$ & $\begin{array}{l}\text { In adults and adolescents aged } \geq 12 \text { years: } \\
\text { - Partial-onset seizures }{ }^{\mathrm{c}} \\
\text { - PGTCS }\end{array}$ & $\begin{array}{l}\text { Adj and Mono } \\
\text { Adj }\end{array}$ \\
\hline
\end{tabular}




\begin{tabular}{|c|c|c|c|c|}
\hline Phenytoin & $\begin{array}{l}\text { Tonic-clonic seizures, partial seizures, or a } \\
\text { combination of these. } \\
\text { Prevention and treatment of seizures following } \\
\text { neurosurgery and/or severe head injury }\end{array}$ & Not specified & $\begin{array}{l}\text { Tonic-clonic seizures and psychomotor (temporal } \\
\text { lobe) seizures. } \\
\text { Prevention and treatment of seizures occurring } \\
\text { during or following neurosurgery }\end{array}$ & Not specified \\
\hline Topiramate & $\begin{array}{l}\text { In patients aged }>6 \text { years } \\
\text { - Partial seizures }{ }^{\mathrm{c}} \text { and } \text { PGTCS } \\
\text { In patients aged } \geq 2 \text { years: } \\
\text { - Partial-onset seizures }{ }^{\mathrm{c}} \text { and PGTCS } \\
\text { - Seizures associated with LGS }\end{array}$ & $\begin{array}{l}\text { Mono } \\
\text { Adj } \\
\text { Adj }\end{array}$ & $\begin{array}{l}\text { In patients aged } \geq 2 \text { years: } \\
\text { - Partial-onset seizures and PGTCS } \\
\text { - Seizures associated with LGS }\end{array}$ & $\begin{array}{l}\text { Adj and Mono } \\
\text { Adj }\end{array}$ \\
\hline Valproate & Generalised, partial or other epilepsy & Not specified & $\begin{array}{l}\text { Complex partial seizures } \\
\text { Simple and complex absence seizures } \\
\text { Patients with multiple seizure types that include } \\
\text { absence seizures }\end{array}$ & $\begin{array}{l}\text { Adj and Mono } \\
\text { Adj and Mono } \\
\text { Adj }\end{array}$ \\
\hline \multicolumn{5}{|c|}{$\begin{array}{l}\text { 'Including tonic-clonic seizures } \\
\text { b Lamotrigine is given as adjunctive therapy but may be the initial AED in LGS. } \\
{ }^{\text {c} W i t h ~ o r ~ w i t h o u t ~ s e c o n d a r y ~ g e n e r a l i s a t i o n ~} \\
\text { AED, antiepileptic drug; Adj, adjunctive; IGE, idiopathic generalised epilepsy; JME, juvenile myoclonic epilepsy; LGS, Lennox-Gastaut syndrome; Mono, monotherapy; } \\
\text { PGTCS, primary generalised tonic-clonic seizures. }\end{array}$} \\
\hline
\end{tabular}




\section{Evidence review}

We reviewed available data on each of these eight AEDs as follows, focussed around five criteria.

\section{Efficacy}

For evidence of AED efficacy, we considered only clinical trials that were submitted to regulatory agencies, as these represent good quality randomised, double blind, placebo-controlled clinical trials. We identified these by matching published studies with those described in the US prescribing information for each AED (we used US prescribing information because insufficient detail of clinical trials is included in UK SPCs). We included trials with extended-release preparations, but excluded any openlabel or uncontrolled studies.

Median percentage reduction in seizure frequency (across entire double-blind period relative to baseline); $50 \%$ responder rate (proportion of patients with $\geq 50 \%$ reduction in seizure frequency in maintenance period relative to baseline); and seizure-freedom rates (during maintenance period) were extracted from published studies and tabulated for each AED and for each seizure type (PGTCS and SGTCS), where available (Table 2). The number needed to treat (NNT) was also calculated for PGTC responder rate and seizure freedom (in keeping with the Consolidated Standards of Reporting Trials (CONSORT) statement [24]). NNT was calculated as 1 over the absolute response rate $(\mathrm{ARR})$, where $\mathrm{ARR}=$ drug responder rate - placebo responder rate. For example, with a placebo responder rate of $49 \%(0.49)$ and a drug responder rate of $72 \%(0.72)$, the NNT is 4.3 - i.e. 4 people need to be treated with drug for 1 person to achieve $\mathrm{a} \geq 50 \%$ reduction in seizure frequency. 


\section{Adverse events}

Reporting of adverse events is incomplete in clinical trial publications (often only reporting events with a frequency of $\geq 5 \%$ or even $\geq 10 \%$ ); therefore, we used SPCs as the source of adverse event data for this review [15-23]. These summaries consist of information validated by the European Medicines Agency to provide healthcare professionals (and public) with evidence-based, reliable and up-to-date information on authorised medicines [25]. They provide data that have been subject to strict and rigorous regulatory scrutiny and are therefore, in our opinion, systematic and unbiased. We report adverse event data in three categories: type A (dose related), type B (idiosyncratic) and type C (teratogenic). For type A (Table 3), we show all adverse events reported at a frequency of greater than 1\%; however, the SPCs for clobazam and phenytoin give no frequency data, so all adverse effects listed in the SPCs were included, unless they were described as rare. For type B events, we have listed rare but important events, extracted from the special warnings and precautions section of the SPCs (Supplementary Table S-1). For type C events, we extracted information, including quantified risk where available, from the fertility, pregnancy and lactation section of SPCs (Supplementary Table S-2).

\section{Drug interactions}

We gathered information from the product SPCs on the metabolic pathways of AEDs, and their impact (inducing or inhibition) on hepatic enzymes (Supplementary Table S3); on the interactions among the eight AEDs (Table 4) and between AEDs and other medications (Supplementary Table S-4).

\section{Adherence}


Many factors can influence adherence [26], and some are easily modifiable by physicians by AED selection and changing dosing regimens. The therapy-related factors that influence adherence to treatment in epilepsy, according to a 2003 WHO report, are: complex treatment regimens, misunderstanding how to take drugs, and adverse events (negative influence); and monotherapy with simple dosing schedules (positive influence) [26]. Lower dosing frequency has been associated with improved adherence in several therapy areas [27,28], including epilepsy [29-31]. We therefore gathered information on dosing frequency (Table 5), titration and maintenance schemes (Supplementary Table S-5), and availability of monotherapy (Table 1), for AEDs approved for PGTC and SG seizures, as factors where treatment choice could directly influence adherence to treatment. We also gathered data on elimination halflife of products (Table 5), as drugs with a long half-life are more able to maintain therapeutic plasma levels in the event of missed doses, than are drugs with shorter half-lives [32].

\section{Mechanism of action}

Information on the pharmacological target(s) of each AED was taken from SPCs (Table 6). In the one case where the SPC did not provide this information directly, we used a recent, independent review article [33]. 


\section{RESULTS}

The information identified by our search methodology is summarised in table format (Tables 1-6, Supplementary Tables S-1-S-5) and elaborated further in the discussion section.

Table 1 shows details of the currently approved indications in Europe and the USA for the eight AEDs that are licenced (as monotherapy or adjunctive therapy) for the treatment of both PGTCS and SGTCS.

\section{Efficacy}

\section{(i) Primary generalised tonic-clonic seizures}

To date, five RCTs in patients with PGTCS have been published: one each for lamotrigine [34], lamotrigine extended release (XR) [35], levetiracetam [36], perampanel [37] and topiramate [38] (Table 2a). These studies provided robust data on efficacy in PGTCS and formed the basis of regulatory approvals in this indication. The RCTs of perampanel and levetiracetam were carefully designed to include patients with PGTCS in the context of idiopathic generalised epilepsy, and the perampanel trial also required confirmation of the IGE diagnosis by an independent review panel [37], whereas the inclusion criteria of the other studies were less stringent. NNT ranges from 3-4 for a response ( $\geq 50 \%$ reduction in seizure frequency) and from 4-13 for seizure freedom (Table 2a). However, the populations in these trials differ substantially so cannot be directly compared.

We found no published RCTs in PGTCS for the four other licensed drugs (carbamazepine, clobazam, phenytoin or valproate). A monotherapy study of clobazam in children has been reported, but this does not distinguish between primary 13 
and secondarily generalised seizures [39]. Carbamazepine, phenytoin and valproate as monotherapy have also been compared in patients with newly diagnosed epilepsy in randomised but non-blinded studies [40], but as these studies did not present separate results for partial and generalised seizures they are not considered here.

(ii) Secondarily generalised tonic-clonic seizures

More RCTs have been published in patients with focal than PGTCS - we identified 17 separate trials for the eight AEDs (Table 2b) [41-57]. However, none of the studies we identified were specifically designed to evaluate SGTCS and were instead powered to detect differences between placebo and study drug for overall focal seizures, not SGTCS. Evaluable data on SGTCS were only available (in primary study publications or secondary publications/reviews) for four AEDs: lamotrigine $[43,44]$, levetiracetam $[45,46]$, perampanel [58] and topiramate [37-42]. Results from these 13 trials are summarised in Table $2 b$.

We found no RCT data for valproate, carbamazepine, phenytoin, or clobazam in SGTCS. Two randomised comparative studies of valproate, phenytoin and carbamazepine in SGTCS (published in 1992 and 1985) did not include comparable endpoints as the other studies we assessed, so are not included in our analysis, but will be briefly mentioned here. In the first study, Mattson et al compared monotherapy with phenytoin, carbamazepine, phenobarbital and primidone in a randomised, double-blind trial in adults with partial-onset seizures. Carbamazepine and phenytoin were similarly effective in controlling SGTCS (at Month 12, $48 \%$ of patients were seizure-free on carbamazepine and $43 \%$ on phenytoin) [59]. In the other study, Mattson et al. compared the effect of valproate and carbamazepine in a randomised, double-blind trial in adults with complex partial or secondarily generalised seizures 
that were untreated or undertreated. Control of SGTCS was similar with both drugs on a variety of measures, and complete seizure control over months 2-12 was achieved in $35 \%$ of patients taking carbamazepine and $31 \%$ of patients taking valproate [60].

Pooled analyses of the regulatory trials of levetiracetam ( $n=3$ trials), perampanel $(n=3)$ and topiramate $(n=5)$ have also been reported. In these pooled analyses, treatment with levetiracetam resulted in a $68 \%$ reduction from baseline in SGTCS frequency compared with a $23 \%$ reduction on placebo [61], perampanel (at $8 \mathrm{mg} /$ day) resulted in a $63 \%$ reduction from baseline in SGTCS frequency compared with a 19\% reduction on placebo [62], and topiramate resulted in a $76 \%$ reduction from baseline in SGTCS frequency compared with a 36\% reduction on placebo [63]. 
Table 2a. Efficacy in PGTC seizures: Data from randomized, placebo-control trials of adjunctive treatment with AEDs approved for

\section{both PGTCS and SGTCS}

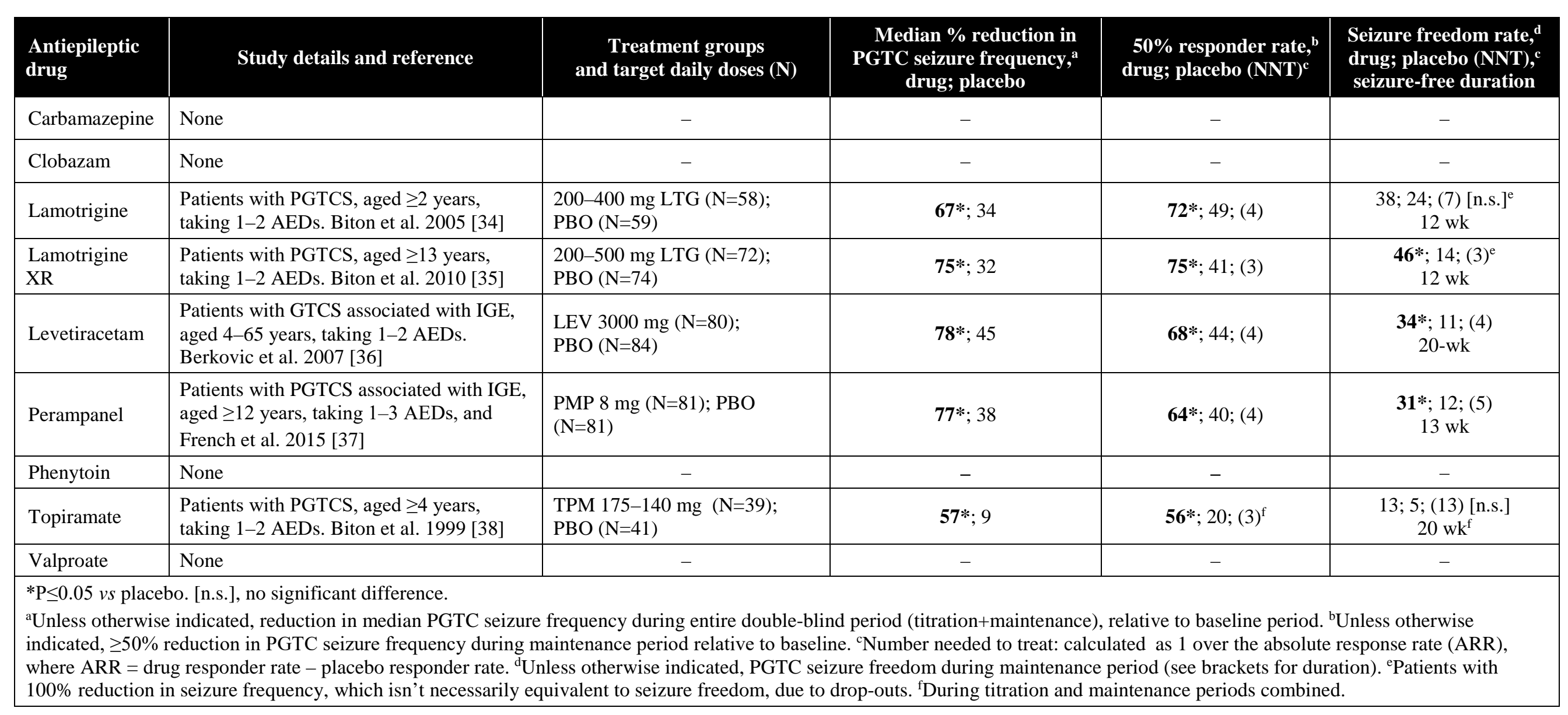


Table 2b. Efficacy in SGTC seizures: Data from randomized, placebo-control trials of adjunctive treatment with AEDs approved for

\section{both PGTCS and SGTCS}

\begin{tabular}{|c|c|c|c|c|c|}
\hline $\begin{array}{l}\text { Antiepileptic } \\
\text { drug }\end{array}$ & Study details and reference & $\begin{array}{l}\text { Treatment groups } \\
\text { and daily doses }(\mathbf{N})\end{array}$ & $\begin{array}{l}\text { Median \% reduction } \\
\text { from baseline in SGS } \\
\text { frequency, }{ }^{\mathrm{a}} \text { drug; placebo }\end{array}$ & $\begin{array}{l}50 \% \text { responder } \\
\text { rate, }^{\text {b drug; placebo }}{ }^{c}\end{array}$ & $\begin{array}{l}\text { SGS freedom rate, } \\
\text { drug; placebo, } \\
\text { seizure-free duration }\end{array}$ \\
\hline Carbamazepine & None & - & - & - & - \\
\hline Clobazam & None & - & - & - & - \\
\hline \multirow{3}{*}{ Lamotrigine } & Matsuo et al. 1993 [41] & \multicolumn{4}{|c|}{$\begin{array}{l}\text { "Only small numbers of patients had secondarily generalized tonic-clonic partial seizures, and no statistical } \\
\text { analysis was performed on this subset." }\end{array}$} \\
\hline & Messenheimer et al. 1994 [42] & \multicolumn{4}{|c|}{$\begin{array}{l}\text { "Less than a third of the study population had secondarily generalized tonic-clonic seizures (GTC), and the } \\
\text { mean monthly frequency for this type of seizure was considerably lower than that observed for simple seizures } \\
\text { and CPS. For these reasons, GTC data were not analyzed separately." }\end{array}$} \\
\hline & $\begin{array}{l}\text { Patients with refractory partial seizures, } \\
\text { aged } 17-63 \text { years, taking } 1-2 \text { AEDs (not } \\
\text { VPA). Schapel et al. } 1993 \text { [43] }\end{array}$ & $\begin{array}{l}\text { LTG } 150-300 \mathrm{mg}(\mathrm{N}=41) \\
\mathrm{PBO}(\mathrm{N}=41) \text {, crossover } \\
\text { design }\end{array}$ & Not reported & $\begin{array}{l}47(\mathrm{~N}=17) ; 16 \\
(\mathrm{~N}=17) \text { [n.t.] }\end{array}$ & Not reported \\
\hline $\begin{array}{l}\text { Lamotrigine } \\
\mathrm{XR}\end{array}$ & $\begin{array}{l}\text { Patients with epilepsy with partial seizures, } \\
\text { aged }>12 \text { years, taking } 1-2 \text { AEDs. } \\
\text { Naritoku et al. } 2007[44]\end{array}$ & $\begin{array}{l}\text { LTG-XR 200-500 mg, } \\
(\mathrm{N}=116) ; \mathrm{PBO}(\mathrm{N}=120)\end{array}$ & $\mathbf{5 5}^{*}(\mathrm{~N}=38) ; 3(\mathrm{~N}=42)$ & $\begin{array}{l}67 *(\mathrm{~N}=38) \\
40(\mathrm{~N}=42)\end{array}$ & Not reported \\
\hline \multirow{3}{*}{ Levetiracetam } & $\begin{array}{l}\text { Patients with uncontrolled partial seizurs, } \\
\text { aged } 16-16 \text { years, taking 1-2 AEDs. } \\
\text { Shorvon et al. } 2000 \text { [45] }\end{array}$ & $\begin{array}{l}\text { LEV } 1000 \mathrm{mg}(\mathrm{N}=106) \\
\text { LEV } 2000 \mathrm{mg}(\mathrm{N}=106) \\
\text { PBO }(\mathrm{N}=112)\end{array}$ & $\begin{array}{c}37(\mathrm{~N}=28) ; 28(\mathrm{~N}=21) \\
-17(\mathrm{~N}=24) \\
{[\text { n.t. }]}\end{array}$ & Not reported & Not reported \\
\hline & $\begin{array}{l}\text { Patients with uncontrolled partial seizures, } \\
\text { aged } 16-70 \text { years, taking } \geq 2 \text { AEDs. } \\
\text { Cereghino et al. } 2000[46]\end{array}$ & $\begin{array}{l}\text { LEV } 1000 \mathrm{mg}(\mathrm{N}=98) ; \mathrm{LEV} \\
3000 \mathrm{mg}(\mathrm{N}=101) ; \mathrm{PBO} \\
(\mathrm{N}=95)\end{array}$ & $\begin{array}{l}\mathbf{8 5}^{*} ; \mathbf{6 5}^{*} ; 24 \\
\text { (Ns not given) }\end{array}$ & Not reported & Not reported \\
\hline & $\begin{array}{l}\text { Patients with partial seizures, aged ,16-70 } \\
\text { years, taking only } 1 \text { AED. Ben Menachem } \\
\text { et al. } 2000 \text { [47] }\end{array}$ & $\begin{array}{l}\text { LEV } 3000 \mathrm{mg}(\mathrm{N}=181) \\
\text { PBO }(\mathrm{N}=105)\end{array}$ & \multicolumn{3}{|c|}{ Outcomes not reported for SG seizures } \\
\hline
\end{tabular}




\begin{tabular}{|c|c|c|c|c|c|}
\hline $\begin{array}{l}\text { Levetiracetam } \\
\text { XR }\end{array}$ & $\begin{array}{l}\text { Patients with partial seizures, aged 12-70 } \\
\text { years, taking 1-3 AEDs. Peltola et al. } 2009\end{array}$ & LEV XR $1000 \mathrm{mg}(\mathrm{N}=79)$; & \multicolumn{3}{|c|}{ Outcomes not reported for SG seizures } \\
\hline \multirow[t]{3}{*}{ Perampanel } & $\begin{array}{l}\text { Patients with partial seizures, aged } \geq 12 \\
\text { years, taking } 1-3 \text { AEDs. Ko and Ramsay, } \\
2013 \text { [58] analysis of Study } 306 \text { [51] }\end{array}$ & $\begin{array}{l}\text { PMP } 2 \mathrm{mg}(\mathrm{N}=180) ; \mathrm{PMP} 4 \\
\mathrm{mg}(\mathrm{N}=172) ; \mathrm{PMP} 8 \mathrm{mg} \\
(\mathrm{N}=169) ; \mathrm{PBO}(\mathrm{N}=185)\end{array}$ & $\begin{array}{r}28(\mathrm{~N}=68) ; 49(\mathrm{~N}=71) ; 69 \\
(\mathrm{~N}=62) ; 36(\mathrm{~N}=69)[\text { n.s.] }\end{array}$ & $\begin{array}{c}44(\mathrm{~N}=68) ; 49 \\
(\mathrm{~N}=71) ; 63(\mathrm{~N}=62) \\
45(\mathrm{~N}=69)[\text { n.s. }]\end{array}$ & $\begin{array}{c}15(\mathrm{~N}=68) ; 23(\mathrm{~N}=71) ; \\
27(\mathrm{~N}=62) ; 24(\mathrm{~N}=69) \\
(13 \text { wk })[\text { n.s.] }\end{array}$ \\
\hline & $\begin{array}{l}\text { Patients with partial seizures, aged } \geq 12 \\
\text { years, taking } 1-3 \text { AEDs. Ko and Ramsay, } \\
2013 \text { [58] analysis of Study } 305[50]\end{array}$ & $\begin{array}{l}\text { PMP } 8 \text { mg }(N=129) ; \text { PMP } \\
12 \text { g }(N=121) ; \text { PBO }(N=136)\end{array}$ & $\begin{array}{c}52 *(\mathrm{~N}=44) ; \mathbf{4 7} *(\mathrm{~N}=43) ; 7 \\
(\mathrm{~N}=48)\end{array}$ & $\begin{array}{c}\mathbf{5 0} *(\mathrm{~N}=44) ; 47 \\
(\mathrm{~N}=43) ; 25(\mathrm{~N}=48)\end{array}$ & $\begin{array}{c}\text { 26*(N=44);16 } \\
(\mathrm{N}=43) ; 5(\mathrm{~N}=48)(13 \\
\text { wk })\end{array}$ \\
\hline & $\begin{array}{l}\text { Patients with partial seizures, aged } \geq 12 \\
\text { years, taking } 1-3 \text { AEDs. Ko and Ramsay, } \\
2013 \text { [58] analysis of Study } 304 \text { [49] }\end{array}$ & $\begin{array}{l}\text { PMP } 8 \text { mg }(\mathrm{N}=133) ; \text { PMP } \\
12 \mathrm{mg}(\mathrm{N}=134) ; \mathrm{PBO} \\
(\mathrm{N}=121)\end{array}$ & $\begin{array}{c}61 *(\mathrm{~N}=51) ; 75^{*}(\mathrm{~N}=52) ; 14 \\
(\mathrm{~N}=56)\end{array}$ & $\begin{array}{c}67 *(\mathrm{~N}=51) ; 60 * \\
(\mathrm{~N}=52) ; 38(\mathrm{~N}=56)\end{array}$ & $\begin{array}{c}\text { 33* }(\mathrm{N}=51) ; 36 * \\
(\mathrm{~N}=52) ; 10(\mathrm{~N}=56)(13 \\
\text { wk })\end{array}$ \\
\hline Phenytoin & None & - & - & - & - \\
\hline \multirow[t]{5}{*}{ Topiramate } & $\begin{array}{l}\text { Patients with partial seizures, aged 18-65 } \\
\text { years, taking 1-2 AEDs. Sharief et al. } \\
1996 \text { [52] }\end{array}$ & $\begin{array}{l}\text { TPM } 400 \mathrm{mg}(\mathrm{N}=23) ; \mathrm{PBO} \\
(\mathrm{N}=24)\end{array}$ & $\begin{array}{c}84(\mathrm{~N}=14) ; 9(\mathrm{~N}=8) \\
\text { [n.t.] }\end{array}$ & $\begin{array}{c}71(\mathrm{~N}=14) ; 38(\mathrm{~N}=8) \\
{\text { [n.t. }]^{\mathrm{d}}}\end{array}$ & $\begin{array}{c}43(\mathrm{~N}=14) ; 25(\mathrm{~N}=8) \\
(11 \text { wk })[\text { n.t. }]^{\mathrm{d}}\end{array}$ \\
\hline & $\begin{array}{l}\text { Patients with refractory partial seizures, } \\
\text { aged } 18-65 \text { years, taking 1-2 AEDs. Ben- } \\
\text { Menachem et al. } 1996 \text { [54] }\end{array}$ & $\begin{array}{l}\text { TPM } 800 \mathrm{mg}(\mathrm{N}=28) ; \mathrm{PBO} \\
(\mathrm{N}=28)\end{array}$ & $\begin{array}{c}90(\mathrm{~N}=11) ; 19(\mathrm{~N}=13) \\
\text { [n.t.] }\end{array}$ & $\begin{array}{l}69(\mathrm{~N}=11) ; 27 \\
(\mathrm{~N}=13)[\text { n.t. }]^{\mathrm{d}}\end{array}$ & $\begin{array}{c}46(\mathrm{~N}=11) ; 18(\mathrm{~N}=13) \\
(13 \text { wk })[\text { n.t. }]^{\mathrm{d}}\end{array}$ \\
\hline & $\begin{array}{l}\text { Patients with refractory partial seizures, } \\
\text { aged } 18-65 \text { years, taking 1-2 AEDs. } \\
\text { Faught et al. } 1996 \text { [55] }\end{array}$ & $\begin{array}{l}\text { TPM } 200 \mathrm{mg}(\mathrm{N}=45) ; \text { ТРM } \\
400 \mathrm{mg}(\mathrm{N}=45) ; \\
\text { TPM } 600 \mathrm{mg}(\mathrm{N}=46) ; \mathrm{PBO} \\
(\mathrm{N}=45)\end{array}$ & $\begin{array}{c}62(\mathrm{~N}=14) ; 100(\mathrm{~N}=15) ; 89 \\
(\mathrm{~N}=13) ; 1(\mathrm{~N}=14) \text { [n.t.] }\end{array}$ & $\begin{array}{c}71(\mathrm{~N}=14) ; 87 \\
(\mathrm{~N}=15) ; 77(\mathrm{~N}=13) \\
21(\mathrm{~N}=14)[\text { n.t. }]^{\mathrm{d}}\end{array}$ & $\begin{array}{c}21(\mathrm{~N}=14) ; 53(\mathrm{~N}=15) ; \\
31(\mathrm{~N}=13) ; 0 \quad(\mathrm{~N}=14) \\
(16 \text { wk })[\text { n.t. }]^{\mathrm{d}, \mathrm{e}}\end{array}$ \\
\hline & $\begin{array}{l}\text { Patients with refractory partial seizures, } \\
\text { aged 18-65 years, taking 1-2 AEDs. } \\
\text { Privitera et al. } 1996 \text { [56] }\end{array}$ & $\begin{array}{l}\text { TPM } 600 \mathrm{mg}(\mathrm{N}=48) ; \text { TPM } \\
800 \mathrm{mg}(\mathrm{N}=48) ; \\
\text { TPM } 1000 \mathrm{mg}(\mathrm{N}=47) ; \\
\text { PBO }(\mathrm{N}=47)\end{array}$ & $\begin{array}{c}66(\mathrm{~N}=12) ; 44(\mathrm{~N}=17) ; 78 \\
(\mathrm{~N}=11) ; 40(\mathrm{~N}=17)[\text { n.t.] }\end{array}$ & $\begin{array}{c}67(\mathrm{~N}=12) ; 47 \\
(\mathrm{~N}=17) ; 55(\mathrm{~N}=11) \\
35(\mathrm{~N}=17)[\text { n.t. }]^{\mathrm{d}}\end{array}$ & Not reported \\
\hline & $\begin{array}{l}\text { Patients with refractory partial seizures. } \\
\text { Tassinari et al., } 1996 \text { [64] }\end{array}$ & $\begin{array}{l}\text { TPM } 600 \mathrm{mg}(\mathrm{N}=30) ; \mathrm{PBO} \\
(\mathrm{N}=30)\end{array}$ & \multicolumn{3}{|c|}{ Outcomes not reported for SG seizures } \\
\hline
\end{tabular}




\begin{tabular}{|c|c|c|c|c|c|}
\hline & $\begin{array}{l}\text { Patients with refractory partial seizures, } \\
\text { aged } 18-65 \text { years, taking CBZ }+/-1 \text { other } \\
\text { AED. Guberman et al. } 2002 \text { [57] }\end{array}$ & $\begin{array}{l}\text { TPM } 200 \mathrm{mg}(\mathrm{N}=171) ; \mathrm{PBO} \\
(\mathrm{N}=92)\end{array}$ & $\mathbf{5 0} *(\mathrm{~N}=55) ; 1(\mathrm{~N}=36)$ & $\begin{array}{c}\mathbf{5 0}^{*}(\mathrm{~N}=55) ; 34 \\
(\mathrm{~N}=36)^{\mathrm{d}}\end{array}$ & Not reported \\
\hline Valproate & None & - & - & - & - \\
\hline \multicolumn{6}{|c|}{ 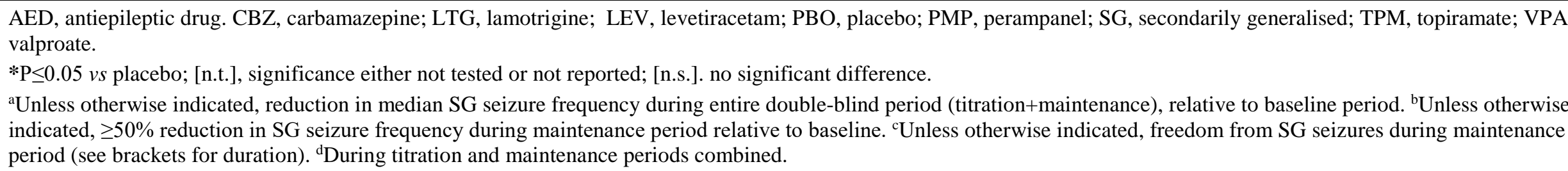 } \\
\hline
\end{tabular}




\section{Adverse effects}

Type A (dose-related) adverse effects

Adverse reactions reported in SPCs at a frequency of $\geq 1 \%$ (common: $1 \%$ to $<10 \%$; very common: $\geq 10 \%$ ) are summarised for each AED in Table 3. Although each AED has a different profile of effects, there was significant overlap.

For clobazam and phenytoin, the frequency of adverse reactions is not given, so all adverse reactions from their SPCs have been listed. We searched for Supplementary clinical trial data for these two AEDs, and found little further detail. Two systematic reviews of data with clobazam as monotherapy [65] and adjunctive therapy [66] for focal or generalised seizures found few studies, and those did not collect or report adverse events in sufficient detail. However, the most common adverse events appear to be drowsiness and dizziness [66]. 
Table 3. "Very common" and "common" adverse drug reactions of the eight approved AEDs, based on information in SPCs

\begin{tabular}{|c|c|c|c|c|c|c|c|c|}
\hline SOC & Carbamazepine $^{a}$ & Clobazam $^{\text {b }}$ & Lamotrigine $^{\mathrm{a}, \mathrm{c}}$ & Levetiracetam $^{a}$ & Perampanel $^{\mathrm{d}}$ & Phenytoin $^{\mathrm{b}}$ & Topiramate $^{a}$ & VPA \\
\hline Blood and lymphatic & $\begin{array}{l}\text { Leukopenia, } \\
\text { thrombocytopenia, } \\
\text { eosinophilia }\end{array}$ & & & & & $\begin{array}{l}\text { Haematoppoietic } \\
\text { complications, }{ }^{\mathrm{e}} \\
\text { lymphadenopathy }^{\mathrm{f}}\end{array}$ & Anaemia & $\begin{array}{l}\text { Anaemia, } \\
\text { thrombocytopenia }\end{array}$ \\
\hline Ear and labyrinth & & & & Vertigo & Vertigo & Vertigo & $\begin{array}{l}\text { Vertigo, tinnitus, ear } \\
\text { pain }\end{array}$ & Deafness $^{g}$ \\
\hline Endocrine & $\begin{array}{l}\text { Oedema, fluid } \\
\text { retention, weight } \\
\text { increase, } \\
\text { hyponatraemia }^{\text {h }} \\
\end{array}$ & & & & & & & \\
\hline Eye & $\begin{array}{l}\text { Accommodation } \\
\text { disorders (e.g., } \\
\text { blurred vision) }\end{array}$ & $\begin{array}{l}\text { Visual disorders } \\
\text { (diplopia, nystagmus) }\end{array}$ & & & $\begin{array}{l}\text { Diplopia, vision } \\
\text { blurred }\end{array}$ & & $\begin{array}{l}\text { Vision blurred, } \\
\text { diplopia, visual } \\
\text { disturbance }\end{array}$ & \\
\hline GI & $\begin{array}{l}\text { Vomiting, nausea, } \\
\text { dry mouth; rectal } \\
\text { irritation }\end{array}$ & $\begin{array}{l}\text { Dry mouth, } \\
\text { constipation, } \\
\text { decreased appetite, } \\
\text { nausea }\end{array}$ & $\begin{array}{l}\text { Nausea, vomiting, } \\
\text { diarrhoea, dry mouth }\end{array}$ & $\begin{array}{l}\text { Abdominal pain, } \\
\text { diarrhoea, dyspepsia, } \\
\text { vomiting, nausea }\end{array}$ & Nausea & $\begin{array}{l}\text { Vomiting, nausea, } \\
\text { gingival hyperplasia, } \\
\text { constipation }\end{array}$ & $\begin{array}{l}\text { Nausea, diarrhoea, } \\
\text { vomiting, } \\
\text { constipation, upper } \\
\text { abdominal pain, } \\
\text { dyspepsia, abdominal } \\
\text { pain, dry mouth, } \\
\text { stomach discomfort, } \\
\text { oral paraesthesia, } \\
\text { gastritis, abdominal } \\
\text { discomfort }\end{array}$ & $\begin{array}{l}\text { Nausea, gastralgia, } \\
\text { diarrhoea }\end{array}$ \\
\hline General & Fatigue & Fall & $\begin{array}{l}\text { Tiredess, pain, back } \\
\text { pain }\end{array}$ & Asthenia/fatigue & $\begin{array}{l}\text { Gait disturbance, } \\
\text { fatigue }\end{array}$ & & $\begin{array}{l}\text { Fatigue, pyrexia, } \\
\text { asthenia, irritability, } \\
\text { gait disturbance, } \\
\text { feeling abnormal, } \\
\text { malaise }\end{array}$ & \\
\hline Hepatobilliary & & & & & & $\begin{array}{l}\text { Acute hepatic failure, } \\
\text { hepatitis toxic, liver } \\
\text { injury }\end{array}$ & & Liver injury \\
\hline Immune system & & & & & & $\begin{array}{l}\text { Anaphylactoid } \\
\text { reaction, } \\
\text { immunoglobulin } \\
\text { abnormalities } \\
\end{array}$ & Hypersensitivity & \\
\hline Infections/infestations & & & & Nasopharyngitis & & & Nasopharyngitis & \\
\hline
\end{tabular}




\begin{tabular}{|c|c|c|c|c|c|c|c|c|}
\hline Injury & & & & & Fall & Fractures & $\begin{array}{l}\text { Arthralgia, muscle } \\
\text { spasms, myalgia, } \\
\text { muscle twitching, } \\
\text { muscular weakness, } \\
\text { musculoskeletal chest } \\
\text { pain }\end{array}$ & \\
\hline Investigations & $\begin{array}{l}\text { GGT increased, } \\
\text { ALP increased }\end{array}$ & & & & Weight increased & & $\begin{array}{l}\text { Weight decreased, } \\
\text { weight increased }\end{array}$ & Weight increased \\
\hline $\begin{array}{l}\text { Metabolism and } \\
\text { nutrition }\end{array}$ & & Weight gain & & Anorexia & $\begin{array}{l}\text { Decreased appetite, } \\
\text { increased appetite }\end{array}$ & $\begin{array}{l}\text { Hypocalcaemia, } \\
\text { hypophosphataemia }\end{array}$ & $\begin{array}{l}\text { Anorexia, decreased } \\
\text { appetite }\end{array}$ & Hyponatraemia \\
\hline $\begin{array}{l}\text { Musculoskeletal and } \\
\text { connective tissue } \\
\text { disorders }\end{array}$ & & & Arthralgia & & Back pain & $\begin{array}{l}\text { Systemic lupus } \\
\text { erythematosus, } \\
\text { arthropathy, decreased } \\
\text { bone mineral density, } \\
\text { osteopenia, } \\
\text { osteoporosis, fractures }\end{array}$ & & \\
\hline $\begin{array}{l}\text { Nervous system } \\
\text { disorders }\end{array}$ & $\begin{array}{l}\text { Ataxia, dizziness, } \\
\text { somnolence, diplopia, } \\
\text { headache }\end{array}$ & $\begin{array}{l}\text { Sedation, fatigue, } \\
\text { sleepiness, slowed } \\
\text { reaction time, } \\
\text { drowsiness, numbed } \\
\text { emotions, confusion, } \\
\text { headaches, dizziness, } \\
\text { muscle weakness, } \\
\text { ataxia, fine tremor, } \\
\text { slowed speech, } \\
\text { unsteady gait, loss of } \\
\text { libido, anterograde } \\
\text { amnesia }\end{array}$ & $\begin{array}{l}\text { Headache, } \\
\text { somnolence, } \\
\text { dizziness, tremor, } \\
\text { insomnia, agitation }\end{array}$ & $\begin{array}{l}\text { Somnolence, } \\
\text { headache, } \\
\text { convulsion, balance } \\
\text { disorder, dizziness, } \\
\text { lethargy, tremor }\end{array}$ & $\begin{array}{l}\text { Dizziness, } \\
\text { somnolence, ataxia, } \\
\text { dysarthria, balance } \\
\text { disorder, irritability }\end{array}$ & $\begin{array}{l}\text { Nystagmus, ataxia, } \\
\text { dysarthria, decreased } \\
\text { coordination, mental } \\
\text { confusion, cerebellar } \\
\text { atrophy, dizziness, } \\
\text { motor twitchings, } \\
\text { headache, } \\
\text { paraesthesia, } \\
\text { peripheral } \\
\text { polyneuropathy, } \\
\text { somnolence, } \\
\text { dysgeusia }\end{array}$ & $\begin{array}{l}\text { Paraesthesia, } \\
\text { somnolence, } \\
\text { dizziness, disturbance } \\
\text { in attention, memory } \\
\text { impairment, amnesia, } \\
\text { cognitive disorder, } \\
\text { mental impairment, } \\
\text { psychomotor skills } \\
\text { impaired, convulsion, } \\
\text { coordination } \\
\text { abnormal, tremor, } \\
\text { lethargy, } \\
\text { hypoaesthesia, } \\
\text { nystagmus, dysgeusia, } \\
\text { balance disorder, } \\
\text { dysarthria, intention } \\
\text { tremor, sedation }\end{array}$ & $\begin{array}{l}\text { Tremor, } \\
\text { extrapyramidal } \\
\text { disorder, stupor, } \\
\text { somnolence, } \\
\text { convulsion, memory } \\
\text { impairment, } \\
\text { headache, nystagmus }\end{array}$ \\
\hline
\end{tabular}




\begin{tabular}{|c|c|c|c|c|c|c|c|c|}
\hline Psychiatric disorders & & $\begin{array}{l}\text { Restlessness, } \\
\text { irritability, insomnia, }{ }^{\mathrm{k}} \\
\text { irritability, acute } \\
\text { agitational states, } \\
\text { anxiety, } \\
\text { aggressiveness, } \\
\text { delusion, fits of rage, } \\
\text { nightmares, } \\
\text { hallucinations, } \\
\text { psychotic reactions, } \\
\text { suicidal tendencies, } \\
\text { frequent musle } \\
\text { spasms, unmasking of } \\
\text { depression, tolerance } \\
\text { and physical } \\
\text { dependence }\end{array}$ & $\begin{array}{l}\text { Aggression, } \\
\text { irritability }\end{array}$ & $\begin{array}{l}\text { Depression, } \\
\text { hostility/aggression, } \\
\text { anxiety, insomnia, } \\
\text { nervousness/ } \\
\text { irritability }\end{array}$ & $\begin{array}{l}\text { Aggression, anger, } \\
\text { anxiety, confusional } \\
\text { state }\end{array}$ & $\begin{array}{l}\text { Insomnia, transient } \\
\text { nervousness }\end{array}$ & $\begin{array}{l}\text { Depression, } \\
\text { bradyphrenia, } \\
\text { insomnia, expressive } \\
\text { language disorder, } \\
\text { anxiety, confusional } \\
\text { state, disorientation, } \\
\text { aggression, mood } \\
\text { altered, agitation, } \\
\text { mood swings, } \\
\text { depressed mood, } \\
\text { anger, abnormal } \\
\text { behaviour }\end{array}$ & $\begin{array}{l}\text { Confusional state, } \\
\text { aggression,' agitation, } \\
\text { disturbance in } \\
\text { attention }^{1}\end{array}$ \\
\hline $\begin{array}{l}\text { Renal and urinary } \\
\text { disorders }\end{array}$ & & & & & & $\begin{array}{l}\text { Tubulointerstitial } \\
\text { nephritis }\end{array}$ & $\begin{array}{l}\text { Nephrolithiasis, } \\
\text { pollakiuria, dysuria }\end{array}$ & \\
\hline Reproductive & & & & & & & & Dysmenorrhoea \\
\hline Respiratory & & $\begin{array}{l}\text { Respiratory } \\
\text { depression }\end{array}$ & & Cough & & Pneumonitis & $\begin{array}{l}\text { Dyspnoea, epistaxis, } \\
\text { nasal congestion, } \\
\text { rhinorrhoea, cough }\end{array}$ & \\
\hline Skin & $\begin{array}{l}\text { Urticaria, (which } \\
\text { may be severe } \\
\text { dermatitis allergic) }\end{array}$ & & ${\text { Skin } \text { rash }^{\mathrm{m}}}$ & Rash & & $\begin{array}{l}\text { Dermatological } \\
\text { manifestations } \\
\text { including } \\
\text { scarlatiniform or } \\
\text { morbilliform rashes }\end{array}$ & $\begin{array}{l}\text { Alopecia, rash, } \\
\text { pruritis }\end{array}$ & $\begin{array}{l}\text { Hypersensitivity, } \\
\text { alopecia (transient/ } \\
\text { dose-related) }\end{array}$ \\
\hline Vascular & & & & & & Polyarteritis nodosa & & Haemorrhage \\
\hline
\end{tabular}


Type B (idiosyncratic) AEs

Rare and serious or potentially serious events extracted from the SPCs for each drug are listed in Supplementary Table S-1.

Type C (teratogenic) AEs

SPCs contain general advice that AED therapy should be reviewed in women who become pregnant or wish to, and discourage the abrupt withdrawal of AEDs. Some warn of the general risk of developmental disorders in mothers with epilepsy. In the lamotrigine and topiramate SPCs, monotherapy is advised whenever possible.

Specific data and recommendations for individual AEDs are listed in Supplementary table S-2. The valproate effects are dose dependant.

\section{Interactions}

Many AEDs interact with hepatic cytochrome P450 enzymes to induce or inhibit their activity, these are summarised in Supplementary Table S-3. The interactions listed in the SPCs between the eight AEDs are summarised in Table 4, and with other drugs in Supplementary Table S-4. 
Table 4. Impact of 'Effector AED' on "Affected AED" plasma levels, according to SPCs, for the eight AEDs approved for treatment of

\section{both PGTCS and SGTCS}

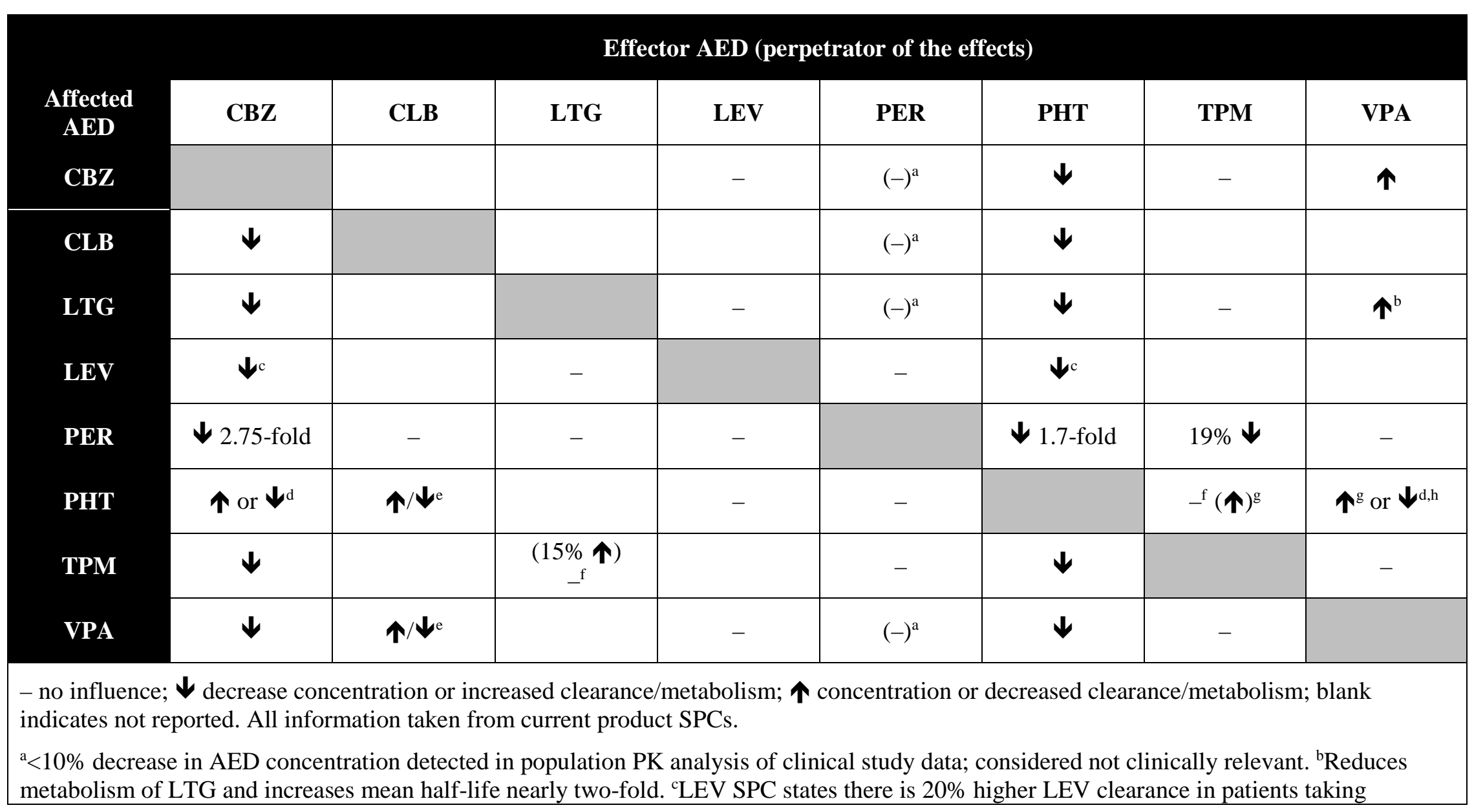




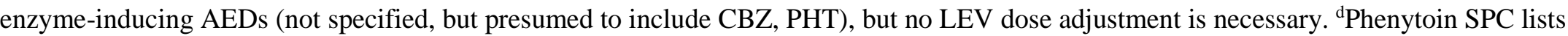
CBZ and VPA as drugs that "may either increase or decrease phenytoin sodium levels." ${ }^{~}$ CLB SPC states that "Addition of clobazam to established anticonvulsant medication (e.g. phenytoin, valproic acid) may cause a change in plasma levels of these drugs." Direction of change

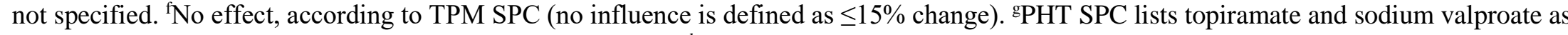
drugs that "may potentially increase phenytoin serum levels." hValproate SPC states that "Epilim decreases phenytoin total plasma

concentration." 


\section{Adherence}

SPC information on AED dosing frequency is shown in Table 5. This is relevant for adherence as drugs with low (once a day) dosing frequency and simple regimens have been associated with improved compliance to long-term therapies in general [26][27,28] and in epilepsy [29-31]. SPC information on half-life is shown in Table 5; the pharmacokinetics of a drug with a long half-life relative to dosing interval mean that is somewhat resistant to the impact of a missed dose [32].

Table 5. SPC-recommended dosing frequency and half-life of AEDs approved

\section{for treatment of both primary and secondarily generalised seizures}

\begin{tabular}{|c|c|c|}
\hline Antiepileptic drug & $\begin{array}{l}\text { Number of } \\
\text { daily doses }^{\mathrm{a}}\end{array}$ & Mean elimination half-life \\
\hline $\begin{array}{l}\text { Carbamazepine } \\
\text { prolonged } \\
\text { release }\end{array}$ & $\begin{array}{c}2-3 \\
2\end{array}$ & $\begin{array}{l}\text { 16-24 hours; with enzyme inducers: } 9-10 \\
\text { hours }\end{array}$ \\
\hline Clobazam & Not stated ${ }^{b}$ & $\begin{array}{l}36 \text { hours (active metabolite } \mathrm{N}- \\
\text { desmethylclobazam, } 79 \text { hours) }\end{array}$ \\
\hline Lamotrigine & $1-2$ & $\begin{array}{l}33 \text { hours (range } 14-103 \text { hours, according to } \\
\text { co-medications) } \\
\sim 14 \text { hours with glucuronidation inducers } \\
\text { (CBZ, PHT) } \\
\sim 70 \text { hours with valproate alone }\end{array}$ \\
\hline Levetiracetam & 2 & $7 \pm 1$ hours \\
\hline Perampanel & 1 & $\begin{array}{l}105 \text { hours (with CYP3A4 inducer CBZ, } 25 \\
\text { hours) }\end{array}$ \\
\hline Phenytoin & $1-3$ & 22 hours (range $7-42$ hours) \\
\hline Topiramate & 2 & 21 hours \\
\hline $\begin{array}{l}\text { Valproate } \\
\text { prolonged } \\
\text { release }\end{array}$ & $\begin{array}{c}2 \\
1-2\end{array}$ & $8-20$ hours \\
\hline \multicolumn{3}{|c|}{$\begin{array}{l}\text { aDosing recommendations taken from the Summary of Product Characteristics for } \\
\text { each AED (usual dosing in adults, oral tablet formulations). } \\
\text { bUsually given 1-2 times daily } \\
\text { AED, antiepileptic drug; CBZ, carbamazepine; CYP, cytochrome P450; PHT, } \\
\text { phenytoin. }\end{array}$} \\
\hline
\end{tabular}




\section{Mechanisms of action}

The main pharmacological targets of the eight AEDs are summarised in Table 6. . These targets can be grouped into four categories based on the known or presumed impact of the AED's binding on synaptic processes and seizure activity. (1) Augmentation of GABA-ergic inhibition (potentiation at $\mathrm{GABA}_{\mathrm{A}}$ receptors, altered GABA synthesis/breakdown). (2) Post-synaptic inhibition of glutamate action (AMPA receptor antagonists). (3) Pre-synaptic inhibition of synaptic neurotransmitter release (SV2A inhibition; P/Q-type voltage-dependent calcium channels). (4) Inhibition of action potential propagation (inhibition of voltage-dependent sodium channels, T-type calcium channels, potentiation of voltage-dependent potassium channels).

Most AEDs have several pharmacological targets and several putative MOAs. Despite valproate having multiple MOAs (affinity for voltage-gated sodium and calcium channels, NMDA receptors, and the GABA system), its most relevant MOA is not yet deciphered. Topiramate also has a wide spectrum of targets. In contrast, carbamazepine and phenytoin exert their actions largely by blocking voltage-gated sodium channels, and this mechanism is thought to be responsible for the apparent worsening of absence and myoclonic seizures observed with these AEDs [67,68]. A single target is also the basis of action of clobazam ( $\mathrm{GABA}_{\mathrm{A}}$ receptors), levetiracetam (SV2A) and perampanel (AMPA receptors). 


\section{Table 6. Primary molecular targets underlying AED mechanisms of action, according to SPCs}

\begin{tabular}{|c|c|c|c|c|c|c|c|c|c|}
\hline \multirow[b]{2}{*}{ Antiepileptic drug } & \multicolumn{3}{|c|}{ Voltage-gated ion channels } & \multicolumn{3}{|c|}{ Glutamate receptors } & \multicolumn{2}{|c|}{$\begin{array}{c}\text { GABA receptors and } \\
\text { turnover }\end{array}$} & \multirow[t]{2}{*}{ Other } \\
\hline & 离 & సँ & $\stackrel{+}{\mathscr{1}}$ & $\sum_{k}^{\ll}$ & 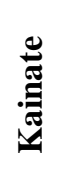 & 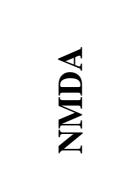 & 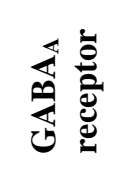 & 苋泀 & \\
\hline Carbamazepine & $\downarrow$ & & & & & & & & $(\mathrm{DA} / \mathrm{NA} \text { turnover })^{\mathrm{a}}$ \\
\hline Clobazam & & & & & & & $\boldsymbol{\top}^{b}$ & & \\
\hline Lamotrigine & $\downarrow$ & & & & & & & & \\
\hline Levetiracetam & & & & & & & & & $\downarrow \mathrm{SV} 2 \mathrm{~A}$ \\
\hline Perampanel & & & & $\downarrow$ & & & & & \\
\hline Phenytoin & $\downarrow$ & & & & & & $(\uparrow)^{\mathrm{c}}$ & & $\left(\right.$ reduce presynaptic $\mathrm{Ca}^{2+}$ entry) ${ }^{\mathrm{c}}$ \\
\hline Topiramate & $\downarrow$ & & & & & & $\uparrow$ & & $(\downarrow \text { carbonic anhydrase })^{\mathrm{a}}$ \\
\hline Valproate & $(\downarrow)^{\mathbf{a}}$ & $(\downarrow)^{\mathbf{a}, \mathbf{d}}$ & & & & $(\downarrow)^{\mathrm{a}, \mathbf{d}}$ & & 个 & \\
\hline \multicolumn{10}{|c|}{$\begin{array}{l}\downarrow \text { inhibitory effect (blockade or inhibition of receptor/ion channel); } \boldsymbol{\uparrow} \text { facilitatory effect; ( ) unclear. } \\
\text { aNot thought by some to contribute to anti-seizure activity [33]. }{ }^{b} \text { No specific target mentioned in SPC. However, clobazam is a benzodiazepine, which all target the GABA } \\
\text { receptor and potentiate the action of GABA on these ion channels [33]. }{ }^{c} \text { Listed in the SPC but not corroborated in a recent expert review article [69]. }{ }^{\mathrm{d} N o t} \text { listed in the SPC but } \\
\text { stated in recent expert reviews [69]. } \\
\text { AED, antiepileptic drug; AMPA, } \alpha \text {-amino-3-hydroxy-5-methyl-4-isoxazolepropionic acid; DA, dopamine; MOA, mechanism of action; NA, noradrenaline; NMDA, N-methyl- } \\
\text { D-aspartate. }\end{array}$} \\
\hline
\end{tabular}




\section{DISCUSSION}

GTCS are a common, serious and potentially life-threatening seizure type, and yet the published literature comprises surprisingly little robust data to inform selection of the most appropriate AED. The factors determining choice of AED for GTCS are too often based on anecdotal information or open-label data of limited validity. In this review, we have considered only published Class 1 studies of efficacy and information from the SPCs, in order to provide a summary of the most robust evidence to guide drug choice. We consider this a key feature of this review. We have included information on the eight drugs that are licensed for use in both PGTCS and SGTCS, and structured our evaluation and summary around five criteria which, in our opinion, are the most relevant considerations for drug selection in the great majority of clinical situations: efficacy, safety, drug interactions, adherence and mechanisms of action. Of these, given the serious nature of this seizure type, efficacy is a particularly important consideration in AED selection for GTCS in our view.

\section{Efficacy}

The approved indications of lamotrigine levetiracetam, perampanel and topiramate are based on the inclusion criteria and results of their regulatory RCTs. In contrast, the older drugs (carbamazepine, clobazam, phenytoin, valproate) have not undergone such rigorous assessment, and the approved indications are generally broader and relatively non-specific (see Table 1), despite their many years of clinical usage. 
For lamotrigine, levetiracetam, perampanel and topiramate, efficacy in PGTCS has been demonstrated in randomized, double-blind, placebocontrolled, parallel-group studies as adjunctive therapy vs placebo. Statistically significant and clinically relevant reductions in PGTC seizure frequency (57-77\%) relative to placebo (9-45\%), and higher responder rates (56-72\% vs 20-29\%) and seizure freedom rates vs placebo (13$48 \%$ vs $5-17 \%$ ) have been reported (Table 2 a) [34-38]. NNT to achieve a response ( $\geq 50 \%$ reduction in seizure frequency) is similar (3-4 for all four AEDs), with a wider variation in the NNT to achieve seizure freedom in these differing patient populations (Table 2a).

Robust data for SGTCS are sparse, as trials generally evaluate changes in overall focal seizure frequency as a primary endpoint, and are typically not powered to evaluate the outcomes related to the (often rarer) SGTCS.

A meta-analysis by Hemery et al. identified 24 RCTs with evaluable data for AEDs in SGTCS (none as a primary endpoint) [70]. Of these trials, 13 provided data (for seven AEDs: lacosamide, perampanel, pregabalin, tiagabine, topiramate, vigabatrin, and zonisamide) for calculation of responder rates and 15 provided data (for seven AEDs: carisbamate, levetiracetam, oxcarbazepine, perampanel, pregabalin, rufinamide, and topiramate) for reliable calculation of the median change in seizure frequency. Only lacosamide, perampanel and topiramate demonstrated statistically superior responder rates $v s$ placebo, and only perampanel, pregabalin and topiramate demonstrated statistically significantly greater reductions in SG seizure frequency $v$ s placebo. The meta-analysis found that confidence intervals overlapped for all drugs, so no AED could be identified as superior to any other. The authors urge caution when interpreting these findings, largely due to methodology and statistical analysis issues, as well as the overriding fact that the studies were not powered to detect effects on SGTCS. No lamotrigine studies were qualified for 
inclusion in this systematic review by Hemery et al. One small cross-over study is mentioned in the lamotrigine USPI, which has data in 17 patients with SGTCS treated with adjunctive lamotrigine [43] and another reports 44 with SGTCS treated with lamotrigine XR [44].

In summary, there is robust evidence of efficacy against PGTCS for four of the licensed AEDs (all in adjunctive use): lamotrigine, levetiracetam, perampanel and topiramate. For the other four licenced drugs (carbamazepine, clobazam, phenytoin and valproate), long usage in clinical practice may imply effectiveness but there are no robust clinical trial data to validate their use. For SGTCS, the evidence is less robust, as SGTCS has not been a primary outcome measure. However, on meta-analysis, there is some evidence of efficacy for the same four AEDs. There is an urgent need for studies specifically designed to assess effects on SGTCS; only then can there be unequivocal evidence of effectiveness and comparative effectiveness in the treatment of this seizure type.

Although no other common AEDs are licensed for GTCS, some physicians do use drugs outside their licensed indications. To do so exposes the prescribing doctor to challenge. If other AEDs are to be used off-label, the physician should clearly explain the rationale to the patient, with the patient should provide explicit consent prior to drug administration.

\section{Adverse effects}


Extrapolating adverse event data from published studies to real clinical situations is difficult, a fact not always been fully appreciated. Data are difficult to evaluate due to the short duration of observation, differences in reporting and classification of adverse events, and the fact that clinical trials are not powered to detect differences in adverse event outcomes. Information from uncontrolled studies and case reports should be interpreted with caution, because of inherent selection biases. For these reasons, we have chosen to use only data provided in the SPCs. These are the data accepted by the regulatory authorities that have as a primary role the protection of patients and the public, and their assessment of adverse effects is in our opinion the most reliable and best authenticated.

\section{Category A: Common dose-related effects}

The adverse effect profiles of drugs differ, and the preferences and tolerances of patients also differ. Therefore, treatment must be tailored to the individual patient, with awareness of what they can expect and what they will tolerate.

Disturbances of vigilance, vestibulocerebellar and motor system: Somnolence, dizziness, blurred vision, incoordination, ataxia and tremor are common side-effects to all AEDs. These can often be managed with knowledge of the pharmacokinetic properties and interactions of AEDs, and sometimes by monitoring blood levels. For example, dizziness with perampanel seems to occur when peak plasma levels are reached (0.5-2.5 $\mathrm{h}$ after administration [71]); therefore if the label dosing instructions to take at bedtime is followed then the impact of dizziness is lessened. 
Disturbances of cognitive abilities: AEDs can cause various cognitive adverse effects - affecting executive function, memory, sustained attention and mental and motor speed [72]. It has been suggested that cognitive side-effects should be monitored routinely in a similar manner to seizure frequency [73]. However, there are very few neuropsychological screening instruments for the reliable assessment of cognition or mood, and some cognitive effects are more related to mood than to cognitive performance [74]. Disentangling the effects of the underlying disease and the AED is a further problem in assessing cognitive and behavioural side-effects.

Psychiatric and behavioural disturbances: These include a range of psychotropic effects (e.g. depression, irritability, aggression, psychosis) that constitute a major factor in limiting the use of some AEDs in specific patients; levetiracetam is particularly problematic in this regard [75] (see recent evidence-based review for more details on epilepsy, aggression, and AEDs [76]). In 2008, based on a meta-analysis of placebocontrolled trials, the US Food and Drug Administration (FDA) issued an alert about an increased risk of suicidal ideation and behaviour associated with AED therapy. This is now reflected as a special warning in the SPCs of all AEDs (Supplementary Table S-1).

The type-A adverse-effect profile often is influential in dictating treatment choice, particularly when epilepsy is easily controlled and the options for drug treatment are broad. As the disease becomes more severe, efficacy often becomes a more important determinant of the AED choice, and patients may accept a higher side-effect load than newly diagnosed patients. This re-emphasises the fact that all treatment is a balance of positive and negative effects and where the balance is drawn is an individual choice. The imperative for the physician is to provide accurate information to the patient on which to base that choice. 


\section{Category B: Idiosyncratic reactions}

Idiosyncratic reactions, sometimes life-threatening, often take years to be recognised because of their rarity. Careful surveillance of new AEDs, and reporting of adverse reactions through the appropriate routes, is therefore crucially important. Most of these events are so uncommon as to not usually influence treatment choice, with the exceptions of rash due to lamotrigine in children and to carbamazepine in certain ethnic groups. Cross-reactivity in relation to allergic skin reactions between carbamazepine, phenytoin and lamotrigine is also relatively common [77]; thus, in patients with a history of allergic skin reactions, it is wise to use AEDs with a low potential for cutaneous reactions (i.e. clobazam, levetiracetam, perampanel, topiramate and valproate).

\section{Category C: Teratogenicity}

GTCS during pregnancy or labour have potentially serious consequences for the foetus and the mother, therefore AED therapy is recommended throughout pregnancy for patients at risk [78]. The SPC advice for each drug is summarised in Supplementary Table S-2. AEDs can induce both congenital malformations and cognitive or behavioural teratogenicity [79], making this an important consideration in the treatment of women of child-bearing potential. Foetal valproate exposure in particular carries a high risk of both congenital malformations and behavioural/cognitive teratogenicity [79]. It is recommended therefore, that valproate "should not be used in female children, female adolescents, or in women of childbearing potential unless alternative treatments are ineffective or not tolerated" and, "The benefit and risk should be carefully reconsidered at regular treatment reviews" [23]. A Joint Task Force of the Commission on European Affairs of the International League Against Epilepsy and 
the European Academy of Neurology has published recommendations on when and how valproate should be used in the treatment of girls and women with epilepsy [80].

Intermediate teratogenic risks exist for phenytoin and topiramate and carbamazepine, whereas lamotrigine and levetiracetam appear to possess low risks for teratogenesis [79]. For clobazam and perampanel, data are still inadequate to estimate the overall risks. Risks seem to increase when multiple AEDs are used, so most recommend using monotherapy where possible. For several AEDs, the risk of major congenital malformations has been shown to be dose related; for valproate monotherapy, frequencies of major congenital malformations at doses of up to about $700 \mathrm{mg} /$ day were consistently substantially lower (5.9\%) than at monotherapy doses of more than $1500 \mathrm{mg} / \mathrm{day}(24.0 \%)$ [81].

Many type-A side-effects are reversible after time or upon dosage adjustment and rarely require treatment discontinuation [82]. For some drugs and adverse effects, sustained-released formulations are preferred for improved tolerability. To minimise the risk of CNS-related adverse effects, it is recommended that AEDs should be slowly up-titrated to the lowest effective maintenance dosage [83-85]. The prescribing doctor should provide accurate, up-to-date information to help each individual patient to balance the risks of each treatment option.

\section{Drug interactions}

Interactions are important to consider in the treatment of generalised seizures, due to the risk of breakthrough seizures if AED blood levels are reduced, and problematic side effects if blood levels are elevated. Most common, are those at the level of the hepatic enzyme systems (Table 4, 
Supplementary Table S-3). As many AEDs are metabolised via cytochrome P450 (CYP) enzymes, they are susceptible to AEDs and other concomitant drugs that inhibit or induce these enzymes. AEDs that are strong inducers or inhibitors of liver enzymes not only impact levels of other AEDs but can affect many other drugs.

Taking all these interactions into account we can distinguish a group of AEDs with critical interactions (phenytoin, carbamazepine, valproic acid, lamotrigine and phenobarbital) and another with fewer critical interactions (clobazam, topiramate, perampanel and levetiracetam. In summary, strategies for minimising interactions should be employed where possible- e.g. the avoidance of polypharmacy, the use of AEDs with minimal interactions, the avoidance of combinations that have major well-documented interactions and the measurement of AED blood levels where appropriate.

\section{Adherence}

Poor adherence to AED treatment is "one of the primary causes of treatment failure" in epilepsy, according to a report from the World Health Organization into the causes of, and solutions to, poor adherence in long-term conditions [26]. Reported non-adherence rates in epilepsy range from $40-60 \%$ in adults, similar to the estimated rate across all long-term conditions (50\% in developed countries) [26]. It can manifest as noninitiation, poor execution (accidental or intentional) or non-persistence, and is affected by many factors related to patient (behaviours, lifestyle), prescribers, socioeconomics, healthcare barriers, and therapy-related factors [26,87-90]. Non-adherence often occurs early after diagnosis and persists over time [91,92]. 
The WHO report gives several recommendations to improve adherence, including spending adequate time with patients, improving patient education around adherence, and reviewing adherence at each visit [26]. Drug choice also plays an important part, and the recommendations include simplifying dosing regimens, reducing dosing frequency, and "linking dose-taking to events in the patient's schedule" [26].

Adherence often has complex multifactorial influences. Side effects also affect adherence, and as well as effective communication between clinician and patient around what to expect, careful AED selection and slow titration may help to reduce the impact of side effects. [64-66]. Dosing frequency has been shown to affect adherence to treatment in people with epilepsy. Compliance rates are highest with once-daily dosing and lowest with 4-times daily dosing [30], the risk of seizures after a missed dose increases by 36\% with each increase in dosing frequency [93], and compliance and satisfaction have been shown to improve with switch from immediate-release valproate to a once daily formulation [29]. Some AEDs have sufficiently long half-lives or have formulations that permit once-daily dosing (Table 5). In summary, selection of AEDs that allow simple dosing regimens (ideally once-daily dosing) that can be tied to patients' regular routines might improve adherence and minimise the potential for break-through generalised seizures, although many factors play a part in this.

\section{Mechanism of action}


Drugs effective in GTCS have various mechanisms of action (MOA) (Table 6). Although no single MOA has been shown to be superior to any other in controlling seizures, MOA should be considered when choosing an AED [94].

Generalized tonic-clonic seizures (GTCS) are sustained by discharges of large neuronal ensembles in the brain, synchronized by brain stem structures; however, different mechanisms underlie PGTCS and SGTCS. PGTCS involves brainstem discharge in which the reticular formation plays a key role, because of its potential to engage a large network and generate 'mass action' under certain circumstances. [95,96], and a key role of AMPA receptors in seizure initiation has been suggested [97]. SGTCS involves propagation of local ictal discharge to brainstem areas to trigger the bilateral tonic-clonic phase. Secondary generalisation is multifactorial, requiring firing of action potentials (via voltage-dependent sodium channels), excitatory synaptic transmission (requiring glutamatergic AMPA receptors), and the failure of inhibitory processes, especially surround inhibition. These multiple processes provide multiple targets and opportunities for controlling seizures spread with AEDs acting via different mechanisms

In patients whose epilepsy is uncontrolled on an initial drug, the practice has evolved of prescribing AEDs with a different MOA. It is rational to substitute an AED that has failed (due to lack of efficacy) with an AED with a different mechanism of action. i.e. from carbamazepine, phenytoin or lamotrigine (sodium-channel blockers) to clobazam (facilitation of GABA signalling), to levetiracetam (inhibition of neurotransmitter release) to perampanel (inhibition of glutamate signalling), or to a mixed AED (valproate or topiramate). And if seizures cannot 
be controlled with monotherapy, mechanism of action is again important in selecting AEDs with complementary targets; or a selective AED with a mixed AED [98-100].

There is reasonable evidence for this 'rational polytherapy' from animal studies, but limited clinical data. One recent retrospective analysis of a claims database showed that patients prescribed AEDs with complementary MOAs persisted on treatment for longer than patients prescribed concomitant AEDs with the same mechanisms (2 GABA-ergic drugs or 2 sodium-channel blockers), and also had reduced inpatient admissions and emergency department visits [101]. No clearly superior combination of mechanisms of action was identified, and effects on seizure frequency could not be quantified.

In summary, MOA is an important consideration in AED selection, and can be a useful part of the patient/physician dialogue. Providing patients with MOA-based rationale for drug options for particular seizure types, or when switching and combining AEDs, can help in the decisionmaking process.

\section{Overall conclusions - drug choice in epilepsy}

So what is the best way to approach treatment of GTCS? It is clear that this dangerous form of seizure must receive optimal therapy. The risks include accidental injury, death, cerebral damage, status epilepticus and social disadvantage, and these risks are likely to sway the risk-benefit balance when making treatment choices. 
There is a good evidence-base showing the efficacy of lamotrigine, levetiracetam, perampanel and topiramate in PGTCS. In the case of SGTCS, the evidence base is poor, and there are no robust data which show conclusive evidence of efficacy, although there is strong suggestive evidence for levetiracetam, perampanel and topiramate. Efficacy must be balanced against the potential side effects, and tailored to the individual patient. For instance, the importance of idiosyncratic effects will depend on the individual's perception of risk, teratogenicity will be relevant only to those who are or may become pregnant, and psychiatric/cognitive side effects are greatly influenced by individual susceptibility. Balance is therefore difficult to achieve and advice requires experience on the part of the physician. Efficacy and safety are the primary considerations, but interactions, adherence, and mechanism of action should also influence decisions. Ideally, AEDs with low potential for interaction, with dosing characteristics that support adherence, and which target logical and complementary seizure mechanisms will be selected.

In modern clinical practice we provide the information, and ultimately the decision about how to strike a balance must be left to the individual patient. Individuals will consider risks differently and will weight side-effects differently. It is the role of the doctor to provide accurate information about the choices.

How much information is required to be imparted is also a difficult question (and one frequently tested in the law courts) but it should include at a minimum the information included in the regulatory patient information leaflet. Fully informed patients are more likely to adhere to treatment and to make wise treatment decisions, and this may lead to a more successful long-term outcome.

\section{ACKNOWLEDGEMENTS}


This paper was the product of a meeting held in Windsor on March 6th 2016. Under direction of the authors, editorial support was funded by

Eisai Europe Ltd. and provided by SuccinctChoice and Kate Carpenter. SDS was supported by the estate of Susan Mary Wright. Dominic Heaney provided important input.

\section{DISCLOSURE OF CONFLICTS OF INTEREST}

SDS has received personal fees from UCB, Sage, Abbott, GSK, Eisai, Sun, and Elsevier; PEB has received personal fees from Eisai, UCB, and Bial; AAG is an employee of Eisai Europe Ltd; GH has received personal fees from Eisai SAS and Advicenne; RK has received personal fees from Eisai, Fenno Medical, GW Pharmaceuticals, Orion, Sage Therapeutics, and Sandoz, Takeda and UCB and educational/research grants from the Academy of Finland, Vaajasalo Foundation, Saastamoinen Foundation, UCB and Eisai.

\section{FUNDING}

Editorial support was funded by Eisai Europe Ltd. 


\section{Abbreviations}

AED, antiepileptic drug; CONSORT, Consolidated Standards of Reporting Trials; EMA, European Medicines Agency; GTCS, generalised tonic-clonic seizure; GCP, Good Clinical Practice; MOA, mechanism of action; NNT, number needed to treat; PGTCS, primary generalised tonic-clonic seizures; RCT, randomised control trial; SGTCS, secondarily generalised tonic-clonic seizures; SPC, Summary of Product Characteristics. 


\section{References}

[1] Gupta S, Kwan P, Faught E, Tsong W, Forsythe A, Ryvlin P. Understanding the burden of idiopathic generalized epilepsy in the United States, Europe, and Brazil: An analysis from the National Health and Wellness Survey. Epilepsy Behav EB 2016;55:146-56. doi:10.1016/j.yebeh.2015.12.018.

[2] Gupta S, Forsythe A, Pomerantz D, Tsong W. The Burden of Primary Generalized Tonic-Clonic Seizures in Europe and the United States: An Analysis of The National Health and Wellness Survey. Value Health J Int Soc Pharmacoeconomics Outcomes Res 2014;17:A402. doi:10.1016/j.jval.2014.08.919.

[3] Sarkis RA, Pietras AC, Cheung A, Baslet G, Dworetzky B. Neuropsychological and psychiatric outcomes in poorly controlled idiopathic generalized epilepsy. Epilepsy Behav EB 2013;28:370-3. doi:10.1016/j.yebeh.2013.05.020.

[4] Taylor RS, Sander JW, Taylor RJ, Baker GA. Predictors of health-related quality of life and costs in adults with epilepsy: a systematic review. Epilepsia 2011;52:2168-80. doi:10.1111/j.1528-1167.2011.03213.x.

[5] Hesdorffer D, Beghi E. ILAE epidemiology commission report: introduction to the supplement. Epilepsia 2011;52 Suppl 7:1.

[6] Hesdorffer DC, Tomson T, Benn E, Sander JW, Nilsson L, Langan Y, et al. Do antiepileptic drugs or generalized tonic-clonic seizure frequency increase SUDEP risk? A combined analysis. Epilepsia 2012;53:249-52. doi:10.1111/j.1528-1167.2011.03354.x.

[7] Monté CPJA, Arends JB a. M, Tan IY, Aldenkamp AP, Limburg M, de Krom MCTFM. Sudden unexpected death in epilepsy patients: Risk factors. A systematic review. Seizure 2007;16:1-7. doi:10.1016/j.seizure.2006.10.002.

[8] Thurman DJ, Logroscino G, Beghi E, Hauser WA, Hesdorffer DC, Newton CR, et al. The burden of premature mortality of epilepsy in highincome countries: A systematic review from the Mortality Task Force of the International League Against Epilepsy. Epilepsia 2017;58:1726. doi:10.1111/epi.13604.

[9] Asadi-Pooya AA, Nikseresht A, Yaghoubi E, Nei M. Physical injuries in patients with epilepsy and their associated risk factors. Seizure 2012;21:165-8. doi:10.1016/j.seizure.2011.10.009.

[10] Nguyen R, Téllez Zenteno JF. Injuries in epilepsy: a review of its prevalence, risk factors, type of injuries and prevention. Neurol Int 2009; 1:e20. doi:10.4081/ni.2009.e20.

[11] Henkin Y, Sadeh M, Kivity S, Shabtai E, Kishon-Rabin L, Gadoth N. Cognitive function in idiopathic generalized epilepsy of childhood. Dev Med Child Neurol 2005;47:126-32.

[12] Kälviäinen R, Salmenperä T, Partanen K, Vainio P, Riekkinen P, Pitkänen A. Recurrent seizures may cause hippocampal damage in temporal lobe epilepsy. Neurology 1998;50:1377-82.

[13] Bernhardt BC, Rozen DA, Worsley KJ, Evans AC, Bernasconi N, Bernasconi A. Thalamo-cortical network pathology in idiopathic generalized epilepsy: insights from MRI-based morphometric correlation analysis. NeuroImage 2009;46:373-81. 
[14] Essential Evidence Plus. Levels of evidence (1a-5) for therapy/prevention/etiology/harms. 2017. https://www.essentialevidenceplus.com/product/ebm_loe.cfm?show=oxford\#.

[15] Carbamazepine SPC. Tegretol Tablets 100mg, 200mg, 400mg. Novartis Pharmaceuticals UK Ltd. 2015. https://www.medicines.org.uk/emc/medicine/1328.

[16] Carbamazepine Prolonged Release SPC. Tegretol Prolonged Release 200mg and 400mg tablets. Novartis Pharmaceuticals UK Ltd. 2016. https://www.medicines.org.uk/emc/medicine/24201.

[17] Clobazam SPC. Frisium 10mg tablets. SANOFI 2017. https://www.medicines.org.uk/emc/medicine/8298.

[18] Lamotrigine SPC. Lamictal. GlaxoSmithKline UK 2017.

[19] Levetiracetam SPC. Keppra 1000mg fillm-coated tablets. UCB Pharma Limited. 2010. https://www.medicines.org.uk/emc/medicine/32704.

[20] Perampanel SPC. Fycompa 2mg, 4mg, 6mg, 8mg, 10mg, 12mg film-coated tablets. Eisai Ltd. 2017. https://www.medicines.org.uk/emc/medicine/26951.

[21] Phenytoin SPC. Epaneutin 30mg/5ml oral suspension. Pfizer Limited. 2017. https://www.medicines.org.uk/emc/medicine/13289.

[22] Topiramate SPC. Topamax 100mg tablets. Janssen-Cilag Ltd. 2017.

[23] Valproate SPC. Epilim 100mg Crushable Tablets. SANOFI 2017. https://www.medicines.org.uk/emc/medicine/23020.

[24] Schulz KF, Altman DG, Moher D, CONSORT Group. CONSORT 2010 statement: updated guidelines for reporting parallel group randomised trials. BMJ 2010;340:c332.

[25] EMA. Summary of Product Characteristics Advisory Group (SmPC AG) 2010-2015 activity report. 2015. http://www.ema.europa.eu/docs/en_GB/document_library/Report/2013/05/WC500143136.pdf.

[26] Sabaté E. WHO report: adherence to long-term therapies. Evidence for action. 2003. http://www.who.int/chp/knowledge/publications/adherence_full_report.pdf.

[27] Saini SD, Schoenfeld P, Kaulback K, Dubinsky MC. Effect of medication dosing frequency on adherence in chronic diseases. Am J Manag Care 2009;15:e22-33.

[28] Laliberté F, Bookhart BK, Nelson WW, Lefebvre P, Schein JR, Rondeau-Leclaire J, et al. Impact of once-daily versus twice-daily dosing frequency on adherence to chronic medications among patients with venous thromboembolism. The Patient 2013;6:213-24. doi:10.1007/s40271-013-0020-5.

[29] Doughty J, Baker GA, Jacoby A, Lavaud V. Compliance and satisfaction with switching from an immediate-release to sustained-release formulation of valproate in people with epilepsy. Epilepsy Behav EB 2003;4:710-6.

[30] Cramer JA, Mattson RH, Prevey ML, Scheyer RD, Ouellette VL. How often is medication taken as prescribed? A novel assessment technique. JAMA 1989;261:3273-7.

[31] Claxton AJ, Cramer J, Pierce C. A systematic review of the associations between dose regimens and medication compliance. Clin Ther 2001;23:1296-310. 
[32] Assawasuwannakit P, Braund R, Duffull SB. Quantification of the Forgiveness of Drugs to Imperfect Adherence. CPT Pharmacomet Syst Pharmacol 2015;4:e0004. doi:10.1002/psp4.4.

[33] Rogawski MA, Löscher W, Rho JM. Mechanisms of Action of Antiseizure Drugs and the Ketogenic Diet. Cold Spring Harb Perspect Med 2016;6. doi:10.1101/cshperspect.a022780.

[34] Biton V, Sackellares JC, Vuong A, Hammer AE, Barrett PS, Messenheimer JA. Double-blind, placebo-controlled study of lamotrigine in primary generalized tonic-clonic seizures. Neurology 2005;65:1737-43. doi:10.1212/01.wnl.0000187118.19221.e4.

[35] Biton V, Di Memmo J, Shukla R, Lee YY, Poverennova I, Demchenko V, et al. Adjunctive lamotrigine XR for primary generalized tonic-clonic seizures in a randomized, placebo-controlled study. Epilepsy Behav EB 2010;19:352-8. doi:10.1016/j.yebeh.2010.07.022.

[36] Berkovic SF, Knowlton RC, Leroy RF, Schiemann J, Falter U, Levetiracetam N01057 Study Group. Placebo-controlled study of levetiracetam in idiopathic generalized epilepsy. Neurology 2007;69:1751-60. doi:10.1212/01.wnl.0000268699.34614.d3.

[37] French JA, Krauss GL, Wechsler RT, Wang X-F, DiVentura B, Brandt C, et al. Perampanel for tonic-clonic seizures in idiopathic generalized epilepsy A randomized trial. Neurology 2015;85:950-7. doi:10.1212/WNL.0000000000001930.

[38] Biton V, Montouris GD, Ritter F, Riviello JJ, Reife R, Lim P, et al. A randomized, placebo-controlled study of topiramate in primary generalized tonic-clonic seizures. Topiramate YTC Study Group. Neurology 1999;52:1330-7.

[39] Clobazam has equivalent efficacy to carbamazepine and phenytoin as monotherapy for childhood epilepsy. Canadian Study Group for Childhood Epilepsy. Epilepsia 1998;39:952-9.

[40] Heller AJ, Chesterman P, Elwes RD, Crawford P, Chadwick D, Johnson AL, et al. Phenobarbitone, phenytoin, carbamazepine, or sodium valproate for newly diagnosed adult epilepsy: a randomised comparative monotherapy trial. J Neurol Neurosurg Psychiatry 1995;58:44-50.

[41] Matsuo F, Bergen D, Faught E, Messenheimer JA, Dren AT, Rudd GD, et al. Placebo-controlled study of the efficacy and safety of lamotrigine in patients with partial seizures. U.S. Lamotrigine Protocol 0.5 Clinical Trial Group. Neurology 1993;43:2284-91.

[42] Messenheimer J, Ramsay RE, Willmore LJ, Leroy RF, Zielinski JJ, Mattson R, et al. Lamotrigine therapy for partial seizures: a multicenter, placebo-controlled, double-blind, cross-over trial. Epilepsia 1994;35:113-21.

[43] Schapel GJ, Beran RG, Vajda FJ, Berkovic SF, Mashford ML, Dunagan FM, et al. Double-blind, placebo controlled, crossover study of lamotrigine in treatment resistant partial seizures. J Neurol Neurosurg Psychiatry 1993;56:448-53.

[44] Naritoku DK, Warnock CR, Messenheimer JA, Borgohain R, Evers S, Guekht AB, et al. Lamotrigine extended-release as adjunctive therapy for partial seizures. Neurology 2007;69:1610-8. doi:10.1212/01.wnl.0000277698.33743.8b.

[45] Shorvon SD, Löwenthal A, Janz D, Bielen E, Loiseau P. Multicenter double-blind, randomized, placebo-controlled trial of levetiracetam as add-on therapy in patients with refractory partial seizures. European Levetiracetam Study Group. Epilepsia 2000;41:1179-86.

[46] Cereghino JJ, Biton V, Abou-Khalil B, Dreifuss F, Gauer LJ, Leppik I. Levetiracetam for partial seizures: results of a double-blind, randomized clinical trial. Neurology 2000;55:236-42. 
[47] Ben-Menachem E, Falter U. Efficacy and tolerability of levetiracetam $3000 \mathrm{mg} / \mathrm{d}$ in patients with refractory partial seizures: a multicenter, double-blind, responder-selected study evaluating monotherapy. European Levetiracetam Study Group. Epilepsia 2000;41:1276-83.

[48] Peltola J, Coetzee C, Jiménez F, Litovchenko T, Ramaratnam S, Zaslavaskiy L, et al. Once-daily extended-release levetiracetam as adjunctive treatment of partial-onset seizures in patients with epilepsy: a double-blind, randomized, placebo-controlled trial. Epilepsia 2009;50:406-14. doi:10.1111/j.1528-1167.2008.01817.x.

[49] French JA, Krauss GL, Biton V, Squillacote D, Yang H, Laurenza A, et al. Adjunctive perampanel for refractory partial-onset seizures: randomized phase III study 304. Neurology 2012;79:589-96. doi:10.1212/WNL.0b013e3182635735.

[50] French JA, Krauss GL, Steinhoff BJ, Squillacote D, Yang H, Kumar D, et al. Evaluation of adjunctive perampanel in patients with refractory partial-onset seizures: results of randomized global phase III study 305. Epilepsia 2013;54:117-25. doi:10.1111/j.15281167.2012.03638.x.

[51] Krauss GL, Serratosa JM, Villanueva V, Endziniene M, Hong Z, French J, et al. Randomized phase III study 306: adjunctive perampanel for refractory partial-onset seizures. Neurology 2012;78:1408-15. doi:10.1212/WNL.0b013e318254473a.

[52] Sharief M, Viteri C, Ben-Menachem E, Weber M, Reife R, Pledger G, et al. Double-blind, placebo-controlled study of topiramate in patients with refractory partial epilepsy. Epilepsy Res 1996;25:217-24.

[53] Topiramate in medically intractable partial epilepsies: double-blind placebo-controlled randomized parallel group trial. Korean Topiramate Study Group. Epilepsia 1999;40:1767-74.

[54] Ben-Menachem E, Henriksen O, Dam M, Mikkelsen M, Schmidt D, Reid S, et al. Double-blind, placebo-controlled trial of topiramate as add-on therapy in patients with refractory partial seizures. Epilepsia 1996;37:539-43.

[55] Faught E, Wilder BJ, Ramsay RE, Reife RA, Kramer LD, Pledger GW, et al. Topiramate placebo-controlled dose-ranging trial in refractory partial epilepsy using 200-, 400-, and 600-mg daily dosages. Topiramate YD Study Group. Neurology 1996;46:1684-90.

[56] Privitera M, Fincham R, Penry J, Reife R, Kramer L, Pledger G, et al. Topiramate placebo-controlled dose-ranging trial in refractory partial epilepsy using 600-, 800-, and 1,000-mg daily dosages. Topiramate YE Study Group. Neurology 1996;46:1678-83.

[57] Guberman A, Neto W, Gassmann-Mayer C, EPAJ-119 Study Group. Low-dose topiramate in adults with treatment-resistant partial-onset seizures. Acta Neurol Scand 2002;106:183-9.

[58] Ko D, Ramsay RE. Perampanel: expanding therapeutic options for patients with medically refractory secondary generalized convulsive seizures. Acta Neurol Scand Suppl 2013;127:36-43. doi:10.1111/ane.12103.

[59] Mattson RH, Cramer JA, Collins JF, Smith DB, Delgado-Escueta AV, Browne TR, et al. Comparison of carbamazepine, phenobarbital, phenytoin, and primidone in partial and secondarily generalized tonic-clonic seizures. N Engl J Med 1985;313:145-51. doi:10.1056/NEJM198507183130303. 
[60] Mattson RH, Cramer JA, Collins JF. A comparison of valproate with carbamazepine for the treatment of complex partial seizures and secondarily generalized tonic-clonic seizures in adults. The Department of Veterans Affairs Epilepsy Cooperative Study No. 264 Group. N Engl J Med 1992;327:765-71. doi:10.1056/NEJM199209103271104.

[61] Leppik IE, Biton V, Sander JWA, Wieser HG. Levetiracetam and partial seizure subtypes: pooled data from three randomized, placebocontrolled trials. Epilepsia 2003;44:1585-7.

[62] Steinhoff BJ, Ben-Menachem E, Ryvlin P, Shorvon S, Kramer L, Satlin A, et al. Efficacy and safety of adjunctive perampanel for the treatment of refractory partial seizures: a pooled analysis of three phase III studies. Epilepsia 2013;54:1481-9. doi:10.1111/epi.12212.

[63] Reife RA, Pledger GW. Topiramate as adjunctive therapy in refractory partial epilepsy: pooled analysis of data from five double-blind, placebo-controlled trials. Epilepsia 1997;38 Suppl 1:S31-33.

[64] Tassinari CA, Michelucci R, Chauvel P, Chodkiewicz J, Shorvon S, Henriksen O, et al. Double-blind, placebo-controlled trial of topiramate (600 mg daily) for the treatment of refractory partial epilepsy. Epilepsia 1996;37:763-8.

[65] Arya R, Anand V, Garg SK, Michael BD. Clobazam monotherapy for partial-onset or generalized-onset seizures. Cochrane Database Syst Rev 2014:CD009258. doi:10.1002/14651858.CD009258.pub2.

[66] Michael B, Marson AG. Clobazam as an add-on in the management of refractory epilepsy. Cochrane Database Syst Rev 2008:CD004154. doi:10.1002/14651858.CD004154.pub4.

[67] Genton P, Gelisse P, Thomas P, Dravet C. Do carbamazepine and phenytoin aggravate juvenile myoclonic epilepsy? Neurology 2000;55:1106-9.

[68] Somerville ER. Some treatments cause seizure aggravation in idiopathic epilepsies (especially absence epilepsy). Epilepsia 2009;50 Suppl 8:31-6. doi:10.1111/j.1528-1167.2009.02233.x.

[69] Löscher W, Klitgaard H, Twyman RE, Schmidt D. New avenues for anti-epileptic drug discovery and development. Nat Rev Drug Discov 2013;12:757-76. doi:10.1038/nrd4126.

[70] Hemery C, Ryvlin P, Rheims S. Prevention of generalized tonic-clonic seizures in refractory focal epilepsy: a meta-analysis. Epilepsia 2014;55:1789-99. doi:10.1111/epi.12765.

[71] Patsalos PN. The clinical pharmacology profile of the new antiepileptic drug perampanel: A novel noncompetitive AMPA receptor antagonist. Epilepsia 2015;56:12-27. doi:10.1111/epi.12865.

[72] Kälviäinen R, Äikiä M, Riekkinen PJ. Cognitive Adverse Effects of Antiepileptic Drugs : Incidence, Mechanisms and Therapeutic Implications. CNS Drugs 1996;5:358-68. doi:10.2165/00023210-199605050-00005.

[73] Witt J-A, Elger CE, Helmstaedter C. Which drug-induced side effects would be tolerated in the prospect of seizure control? Epilepsy Behav EB 2013;29:141-3. doi:10.1016/j.yebeh.2013.07.013.

[74] Marino SE, Meador KJ, Loring DW, Okun MS, Fernandez HH, Fessler AJ, et al. Subjective perception of cognition is related to mood and not performance. Epilepsy Behav EB 2009;14:459-64. doi:10.1016/j.yebeh.2008.12.007. 
[75] Chen Z, Lusicic A, O’Brien TJ, Velakoulis D, Adams SJ, Kwan P. Psychotic disorders induced by antiepileptic drugs in people with epilepsy. Brain J Neurol 2016;139:2668-78. doi:10.1093/brain/aww196.

[76] Brodie MJ, Besag F, Ettinger AB, Mula M, Gobbi G, Comai S, et al. Epilepsy, Antiepileptic Drugs, and Aggression: An Evidence-Based Review. Pharmacol Rev 2016;68:563-602. doi:10.1124/pr.115.012021.

[77] Hirsch LJ, Arif H, Nahm EA, Buchsbaum R, Resor SR, Bazil CW. Cross-sensitivity of skin rashes with antiepileptic drug use. Neurology 2008;71:1527-34. doi:10.1212/01.wnl.0000334295.50403.4c.

[78] Tomson T, Battino D, Bonizzoni E, Craig J, Lindhout D, Perucca E, et al. Withdrawal of valproic acid treatment during pregnancy and seizure outcome: Observations from EURAP. Epilepsia 2016;57:e173-177. doi:10.1111/epi.13437.

[79] Meador KJ, Loring DW, Hulihan JF, Kamin M, Karim R, CAPSS-027 Study Group. Differential cognitive and behavioral effects of topiramate and valproate. Neurology 2003;60:1483-8.

[80] Tomson T. Commentary: Valproate in the treatment of epilepsy in women and girls: The need for recommendations. Epilepsia 2015;56:1004-5. doi:10.1111/epi.13036.

[81] Tomson T, Battino D, Bonizzoni E, Craig J, Lindhout D, Perucca E, et al. Dose-dependent teratogenicity of valproate in mono- and polytherapy: an observational study. Neurology 2015;85:866-72. doi:10.1212/WNL.0000000000001772.

[82] Lee H-W, Jung D-K, Suh C-K, Kwon S-H, Park S-P. Cognitive effects of low-dose topiramate monotherapy in epilepsy patients: A 1year follow-up. Epilepsy Behav 2006;8:736-41. doi:10.1016/j.yebeh.2006.03.006.

[83] Trinka E, Steinhoff BJ, Nikanorova M, Brodie MJ. Perampanel for focal epilepsy: insights from early clinical experience. Acta Neurol Scand 2016;133:160-72. doi:10.1111/ane.12529.

[84] Mula M, Hesdorffer DC, Trimble M, Sander JW. The role of titration schedule of topiramate for the development of depression in patients with epilepsy. Epilepsia 2009;50:1072-6. doi:10.1111/j.1528-1167.2008.01799.x.

[85] Panayiotopoulos CP. Chapter 4: Principles of therapy in epilepsies. Epilepsies Seizures Syndr. Manag., Oxfordshire (UK): Bladon Medical Publishing; 2005.

[86] Brodie MJ, Mintzer S, Pack AM, Gidal BE, Vecht CJ, Schmidt D. Enzyme induction with antiepileptic drugs: cause for concern? Epilepsia 2013;54:11-27. doi:10.1111/j.1528-1167.2012.03671.x.

[87] Brodtkorb E, Samsonsen C, Sund JK, Bråthen G, Helde G, Reimers A. Treatment non-adherence in pseudo-refractory epilepsy. Epilepsy Res 2016;122:1-6. doi:10.1016/j.eplepsyres.2016.02.001.

[88] Gabr WM, Shams MEE. Adherence to medication among outpatient adolescents with epilepsy. Saudi Pharm J SPJ Off Publ Saudi Pharm Soc 2015;23:33-40. doi:10.1016/j.jsps.2014.05.003.

[89] Loiselle K, Rausch JR, Modi AC. Behavioral predictors of medication adherence trajectories among youth with newly diagnosed epilepsy. Epilepsy Behav EB 2015;50:103-7. doi:10.1016/j.yebeh.2015.06.040.

[90] Eatock J, Baker GA. Managing patient adherence and quality of life in epilepsy. Neuropsychiatr Dis Treat 2007;3:117-31. 
[91] Modi AC, Rausch JR, Glauser TA. Patterns of nonadherence to antiepileptic drug therapy in children with newly diagnosed epilepsy. JAMA 2011;305:1669-76. doi:10.1001/jama.2011.506.

[92] Aylward BS, Rausch JR, Modi AC. An examination of 1-year adherence and persistence rates to antiepileptic medication in children with newly diagnosed epilepsy. J Pediatr Psychol 2015;40:66-74. doi:10.1093/jpepsy/jsu010.

[93] Cramer JA, Glassman M, Rienzi V. The relationship between poor medication compliance and seizures. Epilepsy Behav 2002;3:338-42.

[94] Barker-Haliski M, White HS. Glutamatergic Mechanisms Associated with Seizures and Epilepsy. Cold Spring Harb Perspect Med 2015;5:a022863. doi:10.1101/cshperspect.a022863.

[95] Faingold CL. Emergent properties of CNS neuronal networks as targets for pharmacology: application to anticonvulsant drug action. Prog Neurobiol 2004;72:55-85. doi:10.1016/j.pneurobio.2003.11.003.

[96] Ishimoto T, Chiba S, Omori N. Convulsive seizures induced by alpha-amino-3-hydroxy-5-methyl-4-isoxazolepropionic acid microinjection into the mesencephalic reticular formation in rats. Brain Res 2004;1021:69-75. doi:10.1016/j.brainres.2004.03.081.

[97] Yasuda S, Ishida N, Higashiyama A, Morinobu S, Kato N. Characterization of audiogenic-like seizures in naive rats evoked by activation of AMPA and NMDA receptors in the inferior colliculus. Exp Neurol 2000;164:396-406. doi:10.1006/exnr.2000.7401.

[98] Giussani G, Beghi E. Does mechanism of drug action matter to inform rational polytherapy in epilepsy? CNS Neurol Disord Drug Targets 2013;12:426-35.

[99] Kwan P, Schachter SC, Brodie MJ. Drug-resistant epilepsy. N Engl J Med 2011;365:919-26. doi:10.1056/NEJMra1004418.

[100] French JA, Faught E. Rational polytherapy. Epilepsia 2009;50 Suppl 8:63-8. doi:10.1111/j.1528-1167.2009.02238.x.

[101] Margolis JM, Chu B-C, Wang ZJ, Copher R, Cavazos JE. Effectiveness of antiepileptic drug combination therapy for partial-onset seizures based on mechanisms of action. JAMA Neurol 2014;71:985-93. doi:10.1001/jamaneurol.2014.808. 
